\title{
The creeping motion of a spherical particle normal to a deformable interface
}

\author{
By A. S. GELLER, S. H. LEE† AND L. G. LEAL \\ Department of Chemical Engineering, California Institute of Technology, Pasadena, CA 91125
}

(Received 20 September 1985 and in revised form 20 December 1985)

Numerical results are presented for the approach of a rigid sphere normal to a deformable fluid-fluid interface in the velocity range for which inertial effects may be neglected. Both the case of a sphere moving with constant velocity, and that of a sphere moving under the action of a constant non-hydrodynamic body force are considered for several values of the viscosity ratio, density difference and interfacial tension between the two fluids. Two distinct modes of interface deformation are demonstrated : a film drainage mode in which fluid drains away in front of the sphere leaving an ever-thinning film, and a tailing mode where the sphere passes several radii beyond the plane of the initially undeformed interface, while remaining encapsulated by the original surrounding fluid which is connected with its main body by a thin thread-like tail behind the sphere. We consider the influence of the viscosity ratio, density difference, interfacial tension and starting position of the sphere in determining which of these two modes of deformation will occur.

\section{Introduction}

When a fluid droplet or rigid spherical particle moves in one fluid bounded either above or below by a second fluid, the presence of the fluid-fluid interface will affect the motion of the body, and the interface will in turn be deformed by the disturbance flow caused by the sphere or drop. If the particle or drop is moving towards the interface, the body may pass through the interface into the second fluid. Such a process is called coalescence when the body is a drop of the second fluid and breakthrough for a rigid particle or a drop of some third fluid.

The generalized coalescence or breakthrough problem is of interest in many important processes. The stability of a suspension of liquid drops depends primarily on the ability of the drops to resist coalescence. The final separation stages of a liquid-liquid extraction process involve droplets of one liquid, A, rising towards a stationary interface through another liquid, B, and B settling through A (figure 1). The capture of particles by a fibrous mat collector onto which a layer of liquid has condensed is an example of a relevant breakthrough process. A logical problem for initial investigation of the complicated phenomena inherent in these applications is a single rigid sphere moving through a quiescent fluid near a deformable, initially plane interface. Indeed, this problem has already been the subject of intensive study and is commonly identified as 'the' coalescence problem. With one exception, however, (Maru, Wasan \& Kintner 1971), these earlier investigators have assumed that the passage of a particle or drop from one fluid to the other always occurs via the film drainage configuration, depicted in figure 2, with 'film drainage' as the

† Present address: Chevron Oil Field Research Co., La Habra, CA 90631. 


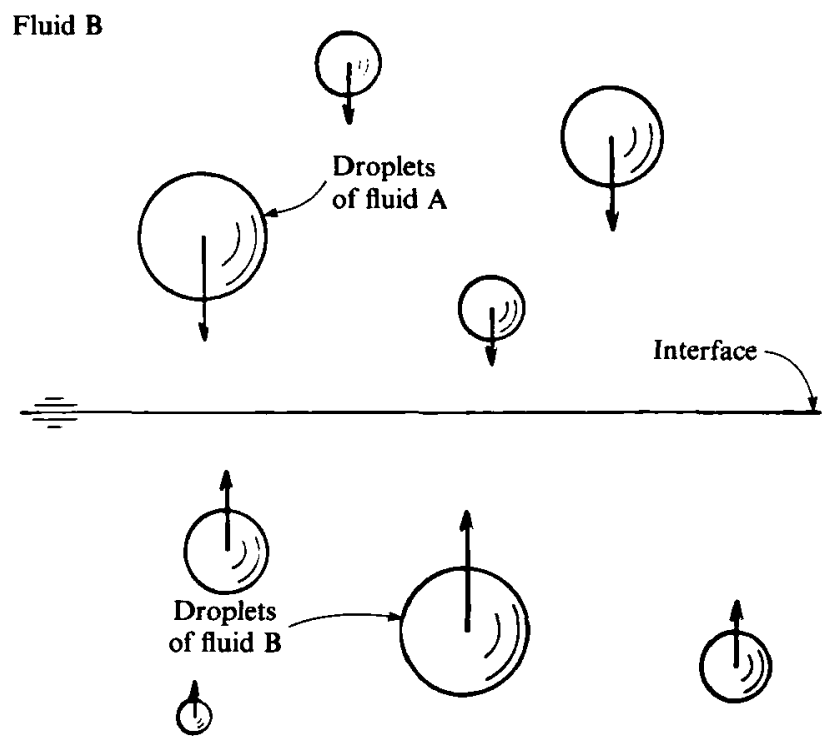

Fluid A

FIGURE 1. Schematic sketch of the final stages of phase separation following liquid-liquid extraction between two immiscible liquids.

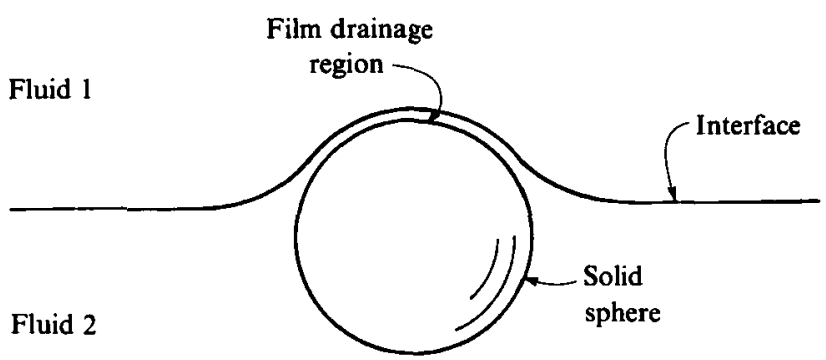

Fiqure 2. Film-drainage configuration for a sphere at a deformable interface.

slowest step in an overall process in which each step can (it is assumed) be studied independently of the others.

In general, however, the prior history of motion affects the instantaneous behaviour of the body and interface so that the total process must be considered from the start and not as individual, independent steps. Indeed, we shall see that consideration of the full initial-value problem of a particle moving towards an interface from 'far' out in one of the fluids shows that the film formation and drainage are not even always steps relevant to the passage of a body from one fluid to another. (See also Leal \& Lee (1981) which contains a preliminary version of some of the material that is reported here.) For certain values of the interfacial tension, density difference and viscosity ratio between the two fluids, a long slender tail may form behind the body as it passes through the original plane of the undisturbed interface, and breakthrough or coalescence could then result from instabilities in this extending thread. The existence of breakthrough by this mode has, in fact, been demonstrated experimentally, but only for a single case of a rigid sphere approaching an initially flat interface at moderate Reynolds number (Maru et al. 1971). 
The emphasis of earlier investigators on the film drainage configuration seems to have resulted from the fact that the sphere or drop used in the early experiments generally had a density either equal to that of the fluid into which it would pass, or between the density of the two fluids. As we shall see, a force balance on a body with density intermediate between the two suspending fluids shows that only a film drainage configuration can result in such a situation. Since most of the time for coalescence or breakthrough in these cases is concentrated in the quasi-static film drainage configuration, most researchers focused their efforts on this portion of the overall process.

A large number of analyses of the film drainage configuration have been reported in the literature. Many of these were reviewed by Jeffreys \& Davies (1971). However, the most satisfactory analysis of the film drainage configuration is that of Jones \& Wilson (1978), who carried out an asymptotic expansion for the sphere position and interface shape using the ratio of gap thickness to sphere radius as the small parameter. This treatment was improved by Smith \& Van de Ven (1984) who also included the effect of gravity on the thin film. The chief shortcoming of previous theoretical work, in our opinion, is the implicit assumption that a draining film will occur in all cases. The only theoretical attempt to explore the possibility of the alternative tail configuration was reported by Maru et al. (1971), but this work contains conceptual errors (see Geller 1986, and $\$ 5$ of this paper).

Experimental data on coalescence and breakthrough at low Reynolds number is surprisingly limited. Studies which tried to investigate the entire coalescence process (Kirkpatric \& Lockett 1974; Narayaran, Gossens \& Kossen 1974) rather than looking at a single step, e.g. film-drainage, have tended to focus on the effects of the disturbance flow caused by drops on each other. A number of very similar film drainage experiments were reported by Princen (1963), Hartland (1969), Maru et al. (1971) and Shah, Wasan \& Kintner (1972).

In these latter experiments, a drop or sphere was held near the interface for some time and then released. This procedure resulted in a narrow gap between the body and interface at the time of release, and yields a film drainage configuration. However, the detailed results may differ substantially from what would occur if the body were released several radii from the undeformed interface. The shape of the interface and the position of the sphere were usually recorded photographically, although Hartland used a capacitance technique to measure the gap thickness. Table 1 shows the conditions in terms of the relevant dimensionless parameters for which experiments have been run with rigid spheres. It is evident that although only a few cases were reported, a fairly wide range of values for the parameters has been covered, albeit with a restricted range of initial conditions.

This paper reports on a numerical study of a rigid sphere moving normal to an initially flat deformable interface subject to one of two conditions on the sphere motion; either the sphere is moving with a constant velocity or it is moving under the action of a constant body force, such as buoyancy. Only gravity, interfacial tension and viscous forces are considered in the force balance on the sphere; the effect of Van der Waal's and other electroviscous forces will be considered in a future study. Earlier work in this research group has examined the case of a sphere moving with constant velocity both normal to the interface (Lee \& Leal 1982) and parallel to it (Berdan 1982) but only under conditions of small or moderate deformation. The present study extends the range of solutions to include large interface deformation for the constant-velocity case, and to obtain corresponding solutions under the condition of constant force which was not considered in the earlier work. Our 


\begin{tabular}{lcccc}
\hline & $\lambda$ & $C a$ & $C g$ & $R e$ \\
Hartland (1968) & 4.76 & $\mathbf{0 . 7 5 3}$ & 0.133 & 3.62 \\
Hartland (1968) & $\mathbf{4 5 . 8 8}$ & $\mathbf{0 . 4 7 0}$ & 0.090 & $\mathbf{3 . 6 2}$ \\
Hartland (1969) & 0.022 & $\mathbf{0 . 4 6 5}$ & 0.089 & 0.003 \\
Hartland (1969) & 0.210 & $\mathbf{0 . 1 5 0}$ & 0.026 & $\mathbf{0 . 0 4 5}$ \\
Maru et al. (1971) & $\mathbf{0 . 4 3 4}$ & $\mathbf{3 . 8 3}$ & $\mathbf{1 . 9 7 2}$ & $\mathbf{1 5 . 1 9}$ \\
Shah et al. (1972) & $\mathbf{0 . 4 4 0}$ & $\mathbf{2 . 5 4 5}$ & $\mathbf{1 . 7 8 2}$ & $\mathbf{8 0 0 . 7 6}$
\end{tabular}

TABLE 1. Published experimental results (photographs or numerical data).

objectives are: to expose those factors which control the details of interface deformation in this purely hydrodynamic problem; to provide a basis for comparison with experimental results so that $(a)$ the influence of non-hydrodynamic factors can be recognized and $(b)$ the effects of such factors as fluid inertia or non-Newtonian rheology can be evaluated; and, finally, in the case of the constant-force problem, to determine conditions for establishment of a tailing configuration, rather than a draining film.

The problem of ultimate interest, of course, is to understand the factors which control the mechanism and dynamics of the breakthrough process. However, at the level of analysis represented by the work reported here, we can investigate this question only in the context of purely hydrodynamic, continuum mechanisms. If, for example, breakthrough should occur by rupture of a thin film due to instabilities associated with London-Van der Waals forces, or even by a purely molecular mechanism where the 'film thickness' is of a molecular (non-continuum) scale, we could not determine that fact in the context of the present theory. Indeed, even if a purely hydrodynamic instability were relevant, its existence might require finer spatial or temporal resolution than is possible with the present methods of solution. What we can determine, however, are the conditions when the combination of viscous, capillary and body forces will lead to a conformation in which a film (or tail) is present. The stability of each configuration must ultimately be treated, including all relevant contributions to the local force balance (i.e. at least electroviscous effects) to judge exactly where and when breakthrough occurs. As indicated above, the effects of Van der Waals and other electroviscous forces will be reported in a future communication.

Let us now turn to the main problem of this paper, namely, the generation of solutions to the full dynamical problem of a sphere moving towards an initially flat, but deforming fluid interface. The mathematical formulation, including the basic governing equations and an outline of the method of solution via the so-called boundary integral technique of low-Reynolds-number hydrodynamics will be presented in the next section. The rest of the paper is concerned with the results and interpretation of our numerical calculations.

\section{Mathematical formulation}

\subsection{Governing equations and boundary conditions}

We consider the translation of a rigid sphere normal to an initially flat but deformable interface between two immiscible, Newtonian fluids. The governing equations and boundary conditions are identical for the sphere falling through the lighter fluid or rising through the heavier fluid; we choose (arbitrarily) to present our analysis and 
results for the latter case. Figure $3(a)$ shows a schematic view of the system for a rising sphere. The analysis which we consider is predicated on the neglect of inertia effects in the fluids and in the motion of the sphere. Thus, we assume

$$
\frac{U_{\infty} a \rho_{2}}{\mu_{2}} \ll 1,
$$

where we have chosen the velocity of the sphere, $U_{\infty}$, in an unbounded fluid 2 as the characteristic velocity, and the radius of the sphere as the characteristic lengthscale. In the constant-velocity formulation of the problem, $U_{\infty}$ is simply the sphere velocity. When the sphere is assumed to move under the action of buoyancy, $U_{\infty}=\frac{2}{9}\left(g a^{2}\left(\rho_{\mathrm{s}}-\rho_{2}\right)\right) / \mu_{2}$ and this is the maximum velocity of the sphere. In this case, (1) is a conservative estimate of conditions necessary for neglect of inertial effects when the sphere is near the interface.

The governing differential equations, in dimensionless form were previously given by Lee \& Leal (1982), and they are simply restated here for convenience

$$
\begin{aligned}
& \left.\begin{array}{l}
0=-\nabla p_{1}+\lambda \nabla^{2} u_{1} \\
0=\boldsymbol{\nabla} \cdot \boldsymbol{u}_{1}
\end{array}\right\} \text { in fluid } 1, \\
& \left.\begin{array}{l}
0=-\nabla p_{2}+\nabla^{2} u_{2} \\
0=\nabla \cdot u_{2}
\end{array}\right\} \quad \text { in fluid 2, } \\
& u_{1}, u_{2} \rightarrow 0 \text { as }|x| \rightarrow \infty,
\end{aligned}
$$

and at the interface $x \in S_{\mathrm{I}}$

$$
\begin{gathered}
u_{1}=u_{2} \\
\lambda \boldsymbol{n} \cdot \boldsymbol{T}_{1}-\boldsymbol{n} \cdot \boldsymbol{T}_{2}=-\frac{1}{C a}(\nabla \cdot \boldsymbol{n}) \boldsymbol{n}+\frac{1}{C g} f \boldsymbol{n} \\
\boldsymbol{n} \cdot u_{1}=\boldsymbol{n} \cdot u_{2}=\frac{1}{|\nabla H|} \frac{\partial f}{\partial t},
\end{gathered}
$$

where the interface shape is denoted as $z=f(r, t)$ and the outward pointing normal $n$ at the interface is $n=\nabla H /|\nabla H|$ with $H \equiv z-f(r, t)$. The stress $T_{i}$ is the total stress minus the hydrostatic contribution and this is the reason why the body-force term appears in the boundary condition (6), rather than in the differential equations (2) and (3). It is convenient for formulation purposes to utilize a cylindrical coordinate system, as indicated in figure $3(a)$, with $z=0$ corresponding to the plane of the underformed interface, and the $z$-axis passing through the centre of sphere at $z=-l(t)$. Three dimensionless parameters result from the non-dimensionalization, the viscosity ratio, $\lambda=\mu_{1} / \mu_{2}$; the capillary number $C a \equiv \mu_{2} U_{\infty} / \gamma$, and $C g \equiv \mu_{2} U_{\infty} / a^{2} g\left(\rho_{2}-\rho_{1}\right)$. The latter two represent ratios of the characteristic viscous stress at the interface relative to surface tension and buoyancy forces, respectively.

The no-slip condition which applies at the sphere surface in all cases is that the fluid velocity equal the velocity of the sphere. In the case of a constant velocity, $U_{\infty}$, this is simply

$$
u_{2}=i_{z} \quad \text { at } x \in S_{\mathrm{P}} .
$$

For the constant-force problem, a force balance on the sphere yields the constraint on the solution

$$
\int_{0}^{\pi} T_{n z}^{\mathrm{P}} \sin \theta \mathrm{d} \theta=3 .
$$

which determines the particle velocity at any instantaneous position. 

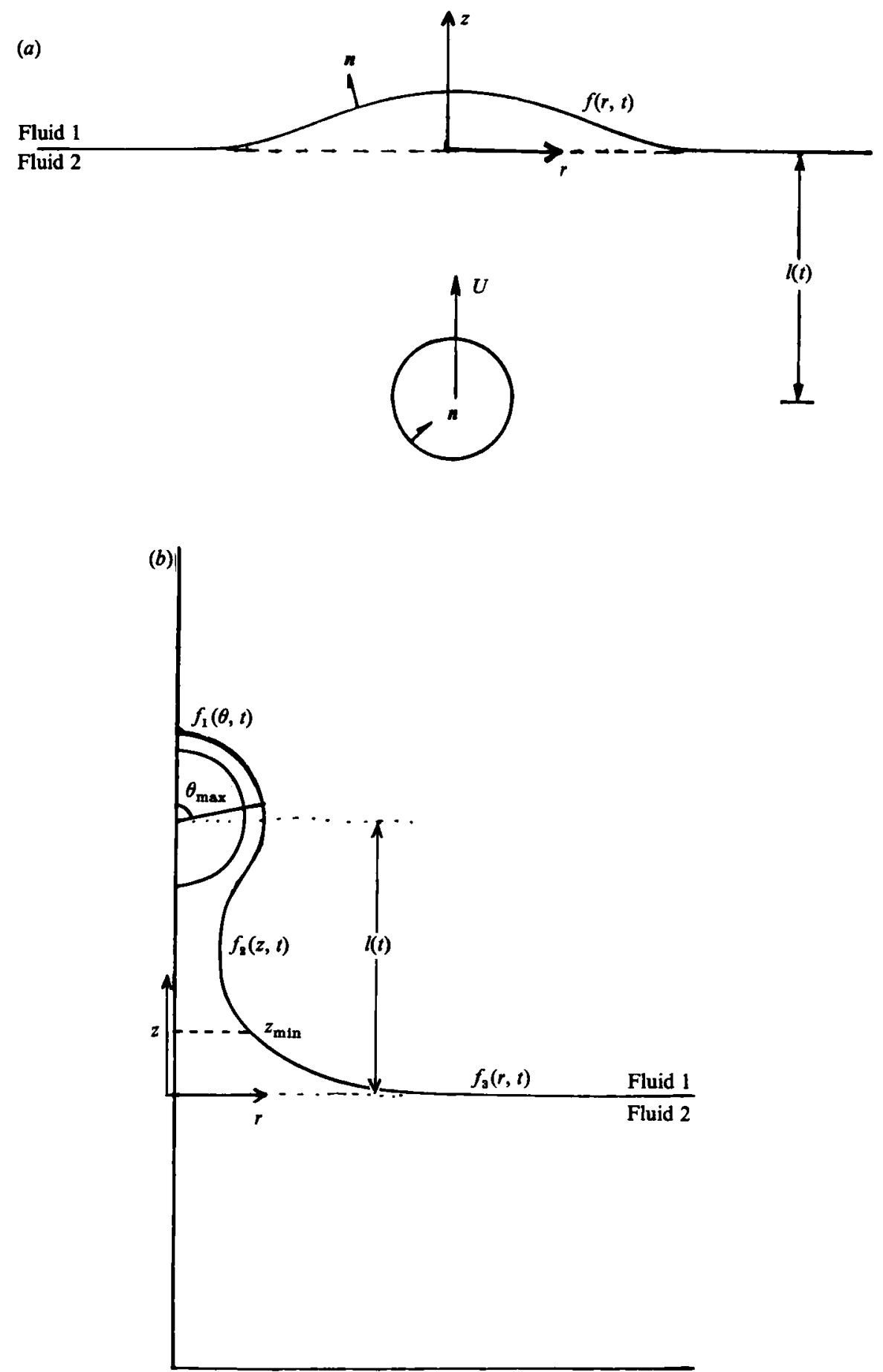

FIGURE 3. (a) Schematic sketch of the system for numerical calculations. (b) The three-function representation of the interface shape. 
The problem then is to solve (2) and (3), subject to the conditions (4)-(8), for the velocity and pressure fields and the interface shape as a function of time and/or particle position.

\subsection{Numerical procedure}

Although the problem in general is nonlinear, the nonlinearity arises from the presence of the unknown shape function $f$, in the boundary conditions; the governing differential equations are themselves linear. Therefore, it is possible to use a fundamental solution for the differential equation, and reduce the calculation to one of finding the particular form of the solution which satisfies the boundary conditions. Following the earlier work of Lee \& Leal (1982), we use the general solution of Stokes' equations due to Ladyzhenskaya (1963) in which point singularities are distributed over the bounding surfaces of the fluid resulting in an integral representation for the pressure and velocity at any arbitrary point in that fluid, $x$ :

$$
\begin{gathered}
u(x)=\frac{1}{8 \pi} \int_{S}\left[\frac{I}{R}+\frac{(x-\eta)(x-\eta)}{R^{3}}\right] \cdot T(\eta) \cdot n d S_{\eta}-\frac{3}{4 \pi} \int_{S} \frac{(x-\eta)(x-\eta)(x-\eta)}{R^{5}} u(\eta) \cdot n d S_{\eta} \\
p(x)=\frac{1}{2 \pi} \int_{S}\left[\frac{1}{R}-\frac{3(x-\eta)(x-\eta)}{R^{5}}\right] \cdot u(\eta) \cdot u \mathrm{~d} S_{\eta}+\frac{1}{4 \pi} \int_{S} \frac{(x-\eta)}{R^{3}} T(\eta) \cdot n S_{\eta},
\end{gathered}
$$

where $\eta=$ position on bounding surface, variable of integration

$$
R \equiv|x-\eta|
$$

Here $S$ represents the boundary of the fluid domain. Thus, when (9) and (10) are applied to fluid $2, S$ includes both the sphere surface and the interface. In fluid 1 , the only boundary is the interface. It will be noted that the weighting functions in the integrals are just the velocity and stress components at the fluid boundaries. Thus, the basic idea is to use the boundary conditions (4)-(8) and the limiting forms of (9) and (10) at the boundaries to obtain a coupled set of integral equations for the boundary velocity and stress components which are unknown. Once these integral equations are solved for boundary values of the velocity and stress, the formulae (9) and (10) can be evaluated to obtain the velocity and pressure at any point in the fluid domain where these quantities are desired. Frequently, however, the only quantities of interest are the particle motion and interface shape and, for this, we need only the surface values of velocity and stress.

The details of applying (9) and (10) together with the boundary conditions at the interface to obtain integral equations for the boundary velocity and stress components have been outlined previously by Lee \& Leal (1982). Here, we content ourselves with merely reporting the results. At the interface we obtain

$$
\begin{aligned}
\frac{1}{2} u^{\mathrm{I}}(x)=-\frac{3}{4 \pi} \int_{S_{\mathrm{I}}} \frac{r r r}{R^{5}} \cdot u^{\mathrm{I}} \cdot n \mathrm{~d} S_{\mathrm{I}}+ & \frac{1}{8 \pi} \int_{S_{\mathrm{I}}}\left[\frac{I}{R}+\frac{r r}{R^{3}}\right] \cdot T_{2}^{\mathrm{I}} \cdot n \mathrm{~d} S_{\mathrm{I}} \\
& +\frac{1}{8 \pi} \int_{S_{\mathrm{P}}}\left[\frac{I}{R}+\frac{r r}{R^{3}}\right] \cdot T_{2}^{\mathrm{P}} \cdot n \mathrm{~d} S_{\mathrm{P}}, \quad x \in S_{\mathrm{I}},
\end{aligned}
$$

and $\quad \frac{1}{2}(\lambda+1) u^{\mathrm{I}}(x)=\frac{3}{4 \pi}(\lambda-1) \int_{S_{1}} \frac{r r r}{R^{5}} u^{\mathrm{I}} \cdot n \mathrm{~d} S_{1}+\frac{1}{8 \pi} \int_{S_{\mathrm{P}}}\left[\frac{I}{R}+\frac{r r}{R^{3}}\right] \cdot T_{2}^{\mathrm{P}} n \mathrm{~d} S_{\mathrm{P}}$

$$
-\frac{1}{8 \pi} \int\left[\frac{I}{R}+\frac{r r}{R^{3}}\right] \cdot F(f) \mathrm{d} S_{\mathrm{I}}, \quad x \in S_{\mathrm{I}}
$$


where the function $F(f)$ is the stress difference at the interface,

$$
\boldsymbol{F}(f) \equiv \lambda \boldsymbol{n} \cdot \boldsymbol{T}_{1}^{\mathrm{I}}-n \boldsymbol{T}_{2}^{\mathrm{I}}=-\frac{1}{C a}\left[\frac{K}{r} \frac{\partial f}{\partial r}+K^{3} \frac{\partial^{2} f}{\partial r^{2}}\right] n+\frac{1}{C g} f n
$$

here expressed in cylindrical coordinates. The superscripts I and $P$ refer to the surface of the interface and sphere, respectively; the vector $r$ is defined as $r \equiv x-\eta$; and $T_{i}^{\mathrm{T}}$ is the stress tensor evaluated as the interface is approached from fluid $i . K$ is defined as $1 /|\nabla H|$ with the shape function $H$ defined in $(15 a, b, c)$. Finally, on the surface of the sphere

$$
\begin{aligned}
u^{\mathrm{P}}(x)=-\frac{3}{4 \pi} \int_{S_{\mathrm{I}}} \frac{r r r}{R^{5}} u^{\mathrm{I}} \cdot n \mathrm{~d} S_{\mathrm{I}}+\frac{1}{8 \pi} \int_{S_{\mathrm{I}}}\left[\frac{I}{R}+\frac{r r}{R^{3}}\right] \cdot T_{2}^{\mathrm{I}} \cdot n \mathrm{~d} S_{\mathrm{I}} \\
+\frac{1}{8 \pi} \int_{S_{\mathrm{P}}}\left[\frac{I}{R}+\frac{r r}{R^{3}}\right] \cdot T_{2}^{\mathrm{P}} \cdot n \mathrm{~d} S_{\mathrm{P}}, \quad x \in S_{\mathrm{P}}
\end{aligned}
$$

Following the precedent of Lee \& Leal (1982), the problem to this point has been described entirely in terms of the cylindrical coordinate system sketched in figure 3(a). Although this representation appears as the 'natural' description in view of the axisymmetric nature of the problem, it can lead to very large values of $\partial f / \partial r$ and $\partial^{2} f / \partial r^{2}$ when the interface approaches a 'tailing' configuration and it was the loss of numerical accuracy associated with this fact which was largely responsible for termination of Lee \& Leal's earlier solutions at relatively modest levels of deformation. In order to overcome this deficiency and ensure maximum numerical accuracy in the calculation of spatial derivatives, we divide the interface into three sections with the interface shape function $H$ represented by a different coordinate system in each section (figure $3 b$ ).

$$
\begin{array}{lll}
H_{1}(x)=\rho-f_{1}(\theta, t)=0, & x \in S_{1}^{\mathrm{I}} & \left(0 \leqslant \theta \leqslant \theta_{\max }\right) \\
H_{2}(x)=r-f_{2}(z, t)=0, & x \in S_{2} & \left(z_{\min } \leqslant z \leqslant z_{\max }\right), \\
H_{3}(x)=z-f_{3}(r, t)=0, & x \in S_{3}^{\mathrm{I}} & \left(r_{\min } \leqslant r\right) .
\end{array}
$$

Equation (15a) locates the interface in terms of the distance from the centre of the sphere as a function of the angle from vertical. In $(15 b)$, the interface shape is expressed in terms of the distance from the $z$-axis as a function of $z$. The 'original' cylindrical coordinate system is used to describe the interface shape as a function of $r$ in $(15 c)$. Although $(15 b)$ and $(15 c)$ are both written in cylindrical coordinates, different functional representations of the interface are used in the two cases. The vector function $F(f)$ is then given by

$$
\begin{aligned}
& F\left(f_{1}\right)=-\frac{1}{C a}\left[\frac{2}{r k_{1}}-\frac{1}{k_{1}^{3} r^{2}} \frac{\partial^{2} f_{1}}{\partial \theta^{2}}-\frac{1}{k_{1} r^{2}} \frac{\partial f_{1}}{\partial \theta} \cot \theta+\frac{1}{k_{1}^{3} r^{3}}\left(\frac{\partial f_{1}}{\partial \theta}\right)^{2}\right] n_{1}+\frac{1}{C g} z n_{1}, \quad \text { for } x \in S_{1}^{\mathrm{I}}, \\
& F\left(f_{2}\right)=-\frac{1}{C a} \frac{1}{k_{2}}\left(\frac{1}{r}-\frac{1}{k_{2}^{2}} \frac{\partial^{2} f}{\partial z^{2}}\right) n_{2}+\frac{1}{C g} z n_{2}, \quad \text { for } x \in S_{2}^{\mathrm{I}}, \\
& F\left(f_{3}\right)=-\frac{1}{C a} \frac{1}{k_{3}}\left(\frac{1}{r} \frac{\partial f}{\partial r}+\frac{1}{k_{3}^{2}} \frac{\partial^{2} f}{\partial r^{2}}\right) n_{3}+\frac{1}{C g} z n_{3}, \quad \text { for } x \in S_{3}^{\mathrm{I}},
\end{aligned}
$$


where

$$
\begin{array}{ll}
k_{1}=\left[1+\frac{1}{r^{2}}\left(\frac{\partial f_{1}}{\partial \theta}\right)^{2}\right]^{\frac{1}{2}}, & n_{1}=\frac{1}{k_{1}}\left(i_{r}-\frac{1}{r} \frac{\partial f_{1}}{\partial \theta} i_{\theta}\right), \\
k_{2}=\left[1+\left(\frac{\partial f_{2}}{\partial z}\right)^{2}\right]^{\frac{1}{2}}, & n_{2}=\frac{1}{k_{2}}\left(i_{r}-\frac{\partial f_{2}}{\partial z} i_{z}\right), \\
k_{3}=\left[1+\left(\frac{\partial f_{3}}{\partial r}\right)^{2}\right]^{\frac{1}{2}}, & n_{3}=\frac{1}{k_{3}}\left(i_{z}-\frac{\partial f_{3}}{\partial r} i_{r}\right) .
\end{array}
$$

The derivatives in the equation for the normal stress jump junction $F(f)$ were evaluated by use of a cubic spline polynomial. A spline function was fit through the centre points of the interface segments in each of the three regions and differentiated analytically at the node points to give $\partial f_{i} / \partial x_{i}\left(x_{i}=\theta, z, r\right)$. The spline function was also used to prevent the node points from convecting along the interface as the calculation progressed so that the original segment distribution was maintained throughout a numerical run.

When the sphere velocity is known so that (8a) applies, (11), (12) and (14) give three integral equations for the unknown quantities $\boldsymbol{u}^{\mathrm{I}}, \boldsymbol{T}_{2}^{\mathrm{I}}$ and $\boldsymbol{T}^{\mathbf{P}}$, provided the interface shape and sphere position are known. When we consider the constant-force problem, on the other hand, $(8 b)$ applies and we have four integral equations for $\boldsymbol{u}^{\mathrm{I}}$, $\boldsymbol{u}^{P}, \boldsymbol{T}_{2}^{\mathrm{T}}$ and $\boldsymbol{T}^{\mathrm{P}}$. Having solved these equations in either case for some specified initial shape, the kinematic condition, (7), can be used to calculate $\partial f / \partial t$. This allows the interface shape to be determined at some later time by adding $\partial f / \partial t \Delta t$ to the present shape. The position of the sphere is also changed by an increment $u^{\mathrm{P}} \cdot \Delta t$ and the process is repeated using the new sphere position and interface shape. In this way, the sphere is 'marched' forward from any given initial condition.

As the problem of motion normal to the interface is axisymmetric, the surface integrals can be simplified greatly by analytically integrating in the azimuthal direction to reduce the surface integrals to line integrals. Equations (11), (12) and (14) then yield seven linear integral equations in the seven unknowns $u_{r}^{\mathrm{I}}, u_{z}^{\mathrm{I}}, u_{z}^{\mathbf{P}}, T_{2 n z}^{\mathrm{I}}$, $T_{2 n r}^{\mathrm{I}}, T_{n z}^{\mathrm{P}}, T_{n r}^{\mathrm{P}}$. Following the work of Lee \& Leal (1982), these equations were solved numerically using a simple collocation method. This technique converts the integral equations into a system of linear algebraic equations. The surface of the sphere and interface were divided into segments small enough that $\boldsymbol{u}^{I}, \boldsymbol{u}^{\mathbf{P}}, \boldsymbol{T}_{2}^{I}$ and $\boldsymbol{T}^{\mathbf{P}}$ could be approximated throughout the segment by the value at the centre of the segment. The criteria used here to determine the size of these segments is discussed in the following section. The integrations were then carried out by Simpson's rule. This procedure converts the seven integral equations into a system of $\left(4 N_{I}+2 N_{\mathrm{P}}+1\right)$ linear algebraic equations where $N_{\mathrm{I}}$ is the number of segments on the interface and $N_{\mathrm{P}}$ is the number of segments on the sphere. This system is readily solved by Gaussian elimination. One complication is that the integrands in (11), (12) and (14) become singular when $x \rightarrow \eta$ (see (9) for definitions of $\boldsymbol{x}$ and $\eta$ ). Thus integration over a small neighbourhood of $x$ was carried out analytically using a linear expansion of the integrands in (11)-(14) so that all terms remain bounded during the numerical integration.

The solutions generated by this numerical scheme are time-dependent through the kinematic condition (7), although the governing equations for the fluids are the steady Stokes equations. All of the equations and boundary conditions (2)-(7) are based upon the assumption of a characteristic timescale

$$
t_{\mathrm{c}}=\frac{a}{U_{\infty}} .
$$


Thus, any motion, including a time-dependent instability, which evolves on a timescale of this order or slower will be resolved by the numerical-solution scheme. Surface-tension-driven flows, with a lengthscale $l$, will be characterized by a timescale

$$
t^{*}=\frac{\mu_{2} l}{\sigma}
$$

Hence, such motions will be resolved if

$$
o\left(\frac{\mu_{2} l}{\sigma}\right) \geqslant O\left(\frac{a}{U_{\infty}}\right) .
$$

This condition can be written in the alternative form

$$
\frac{l}{a} C a \geqslant O(1)
$$

Thus, any surface-tension-driven disturbance with a lengthscale $l=O(a)$ or larger will be resolved for systems with $C a=O(1)$. Very small wavelength disturbances, or disturbances in systems with a large interfacial tension will not be resolved.

\section{Preliminary results}

The solution to the problem formulated in the previous section will generally depend on both the initial location of the sphere and the initial shape of the interface. Therefore, a new set of calculations is required, in principle, for every combination of initial conditions. In an attempt to circumvent this considerable complication, Lee \& Leal (1982) pursued the concept of 'limiting solutions' in which the characteristic time for displacement of the sphere is either very much larger or very much smaller than an intrinsic measure of the characteristic timescale for interface motion. These two cases correspond to an interface that remains flat for the fast-moving sphere, or one which reaches a steady-state deformation, i.e. $\boldsymbol{u} \cdot \boldsymbol{n}=0$ at each instantaneous position of the sphere in the case of 'slow' transition. In either of these cases, the velocity of the sphere and the interface shape (or velocity) would depend only on $\lambda$, $\mathrm{Ca}, \mathrm{Cg}$ and the instantaneous position of the sphere, thereby removing the prior history of sphere motion and interface shape as a factor in the solution. Unfortunately, comparison with exact numerical solutions showed that only relatively few combinations of $\lambda, C a, C g$ and initial sphere position corresponded accurately to these limiting cases.

In the present paper, we have therefore considered further the dependence of the solution on the initial position of the sphere. It is intuitively evident that a starting position which is sufficiently large should have a small effect on the solution when the sphere is near the interface. This is illustrated in figures 18 and 20 for results obtained under the conditions of a constant force, $(8 b)$. In these figures, interface shapes are plotted in a reference frame moving with the sphere so that the interface appears to be sweeping past the sphere for starting positions, $l_{0}$, of 3,5 and 10 radii from an undeformed interface, with $\lambda=1, C a=1, C g=1$ and $\lambda=10, C a=1$, $C g=1$. We shall discuss these results in some detail in $\$ 5$. For present purposes, it is sufficient to note that the interface shapes for starting positions of 5 and 10 appear to be identical by the time the sphere reaches a position 3 radii away from the original interface position. The case starting at 3 also produces deformation equal to the other two cases once the sphere reaches $l=0.75$. Figure 20 indicates that for a higher viscosity ratio, it takes longer for the three cases to converge to the same shape, but 


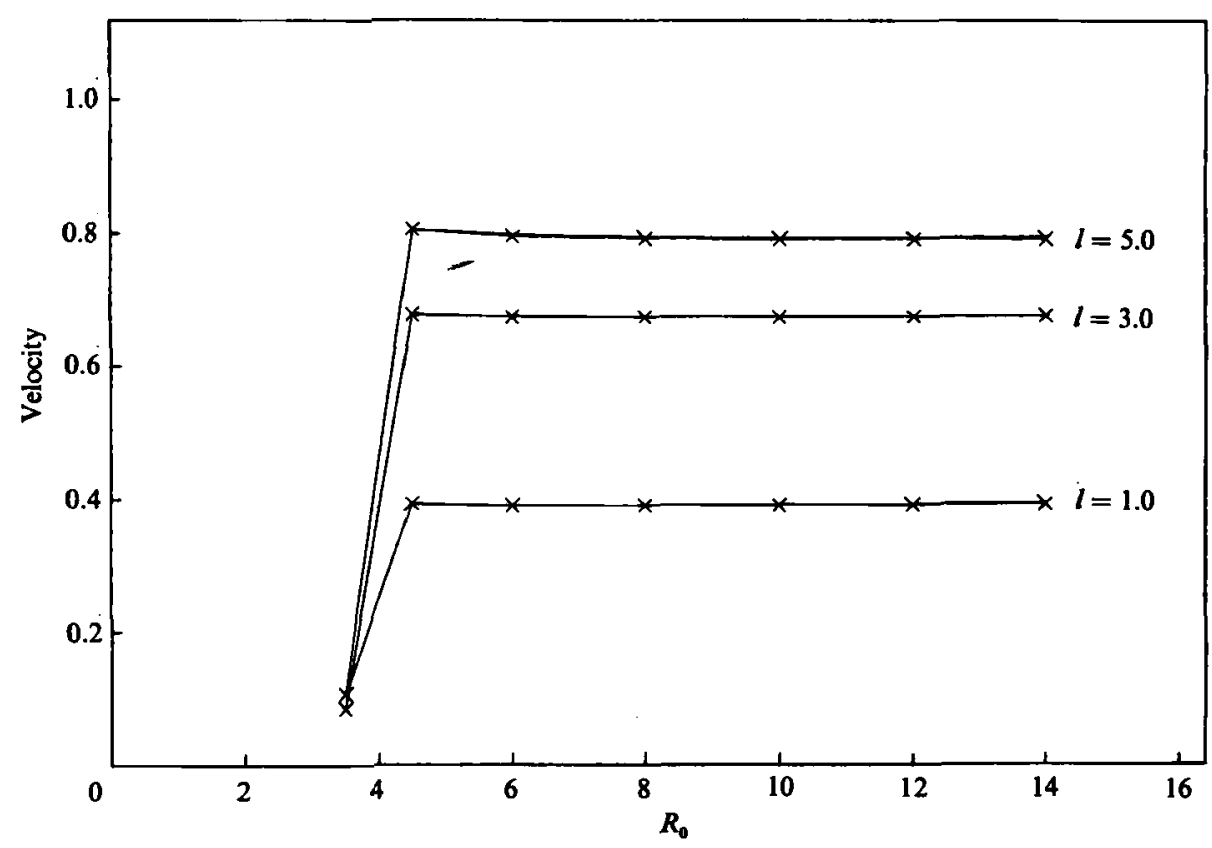

Figure 4. Sphere velocity as a function of $R_{0}$ for the limiting case of slow sphere motion (i.e. $\left.u^{\mathrm{I}} \cdot n=0\right) . l=1.0,3.0,5.0 ; \lambda=10, C a=C g=1$ for the constant force problem ( $\times$, results from calculations).

after $l=0.10$, the results for the various starting positions are indistinguishable. Plots of sphere velocity versus position for these cases are given in figures 19 and 21 . Again, the results for the three cases converge as the sphere reaches a position straddling the interface. Finally, figure 5 gives the same type of plot for the constant-velocity case with $\lambda=1, C a=1, C g=1$ with the same qualitative results. It is evident that a starting position $l_{0}=3$ is large enough to produce solutions which are qualitatively (and over most of the trajectory quantitatively) representative of the solution for a sphere approaching an interface from any large distance, and we use the value $l_{0}=3$ for many of the computations that are reported in this paper. Solutions with a smaller initial separation between the sphere and the interface can, however, exhibit important qualitative differences from these 'large initial separation' solutions, as we shall consider later in some detail.

Another aspect of the numerical calculation which should be discussed prior to the detailed results is the fact that the domain of numerical integration along the interface in (13), (14) and (16) is necessarily truncated at some finite distance $R_{0}$, from the centreline. Numerical and asymptotic justification for this procedure was provided by Lee \& Leal (1982) for the case of sphere motion at a constant velocity. Figure 4 demonstrates the effect of increasing $R_{0}$ on the sphere velocity for the constant-force problem at three different sphere positions. All other calculations in this study were carried out with $R_{0} \geqslant 12$. It may also be noted that the truncation is equivalent to assuming $\boldsymbol{u}^{\mathrm{I}}$ and $\boldsymbol{T}^{\mathrm{I}}=0$ for $r>R_{0}$. Thus, additional justification of the truncation procedure can be achieved by comparing the calculated values of $\boldsymbol{u}^{\mathrm{I}}$ and $\boldsymbol{T}^{I}$ near the point of truncation with values near the centreline. Generally, several orders of magnitude difference existed between these two sets of values.

Although it is a fairly straightforward procedure to decide on a reasonable lower 
bound for $\boldsymbol{R}_{0}$, deciding on the segment sizes along the sphere and interface surfaces is a more complex problem. Of course, placing more segments on the surface comes closer to representing the process of integration, but adding segments to the surface increases computation time and cost. The number of segments necessary is strongly influenced by the details of the weighting function distribution along the surface. In the collocation method used here, it is assumed that $\boldsymbol{u}^{\mathrm{I}}$ and $\boldsymbol{T}^{\mathrm{I}}$ or $\boldsymbol{T}^{\mathrm{P}}$ are constant at the centre-point value within each segment; thus, the density of segments must be largest in regions where the values of these variables change most rapidly. Also, the contribution from any segment decreases at least as fast as $1 / r^{2}$ as $r$ increases, so larger segment increments can be used as the distance along the interface increases. The solutions are axisymmetric and we have already indicated that the azimuthal angle $\phi$ has been integrated out from the full integral equations (13)-(16) to obtain the equations which are solved here. Thus the collocation 'segments' on the sphere are actually 'strips' around the sphere, with the sphere surface incremented in terms of the polar angle $\theta$. Following Lee \& Leal (1982) a uniform segment distribution in $\theta$ was chosen for the sphere, with 10 increments in $\theta$ covering the sphere surface when the interface deformation was small or moderate, increasing to 16 for large deformations and/or small sphere-interface separations.

A non-uniform distribution of segment sizes was used along the interface. The number of segments (which again are strips encircling the $(r=0)$-axis) was taken as 14 for 'stiff' interfaces, i.e. those that showed slight deformation, and 16-35 for interfaces with large deformation, again the number increasing as the deformation grew.

When the three-coordinate-system representation was used to describe the interface, two additional parameters were specified, $\Theta_{\max }$ and $z_{\min }$. These were chosen to correspond to the end of the spherical cap and tail regions, respectively, as illustrated qualitatively in figure $3(b)$. As a result, in each of the three basic regions the interface closely followed a coordinate surface except in the area where one representation changed over into another, and the partial derivatives of the shape functions in each region had small magnitudes in the chosen coordinate representation.

The final parameter of the numerical algorithm was the timestep used in incrementing the interface shape and sphere position. This marching was carried out by simply multiplying the instantaneous velocity of the sphere or interface segment, calculated by the collocation technique, by the timestep. In other words, the exact equation for sphere position,

$$
l=l_{0}+\int_{t_{0}}^{t} u^{\mathbf{P}} \mathrm{d} t
$$

is approximated by,

$$
l=l_{0}+\sum_{i=1}^{n} u_{i}^{\mathrm{P}} \Delta t_{i}
$$

where a new $u_{i}^{\mathrm{P}}$ is calculated at each timestep, and similarly for the interface. The largest non-dimensional timestep, $\Delta t=\Delta t^{\prime} /\left(a / U_{\infty}\right)$ used was 0.05 and this value was decreased as the rate of change of the sphere position or interface shape increased. Also, for both constant-force and constant-velocity calculations, a shorter timestep is needed as the distance between two surfaces (for example the sphere and the interface) decreases. Although this time-marching procedure is accurate only to $O(\Delta t)$, the requirement that $\Delta t$ decrease as bounding surfaces approach necessitates a small enough timestep that it is not necessary to employ higher-order methods. Whenever a new timestep was introduced, an overlap region with the old larger timestep was included in the calculation. The shapes and velocities in this region were compared 
for the two cases and if the differences had been greater than $2 \%$, the small timestep would have been introduced at an earlier point until the difference was within $2 \%$. In all cases, however, the difference actually computed was less than $0.5 \%$ when the new timestep was introduced.

\section{Results for motion of a sphere from a large distance at constant velocity}

The numerical scheme described in the preceding two sections has been used to investigate the motion of a sphere normal to a deformable interface. We first consider the case where the sphere moves with a constant velocity from a large distance. The results presented in this section complement Lee \& Leal (1982) who considered the same problem but were limited to calculations for moderate deformation only. Using the three-coordinate-system representation, we have now been able to numerically consider cases where the interface shows large deformation, continuing in some cases to the point where the sphere has passed many radii beyond the plane of the undeformed interface. A feature of the numerical algorithm is that the maximum allowable timestep becomes smaller as the thickness of either the film of liquid in front of the sphere or the tail becomes thinner. Thus, the present computations were terminated when the cost to further increment the sphere position by a small distance became unacceptably large. In no case was this computational termination point coincident with the onset of instability of the film or tail, or of 'contact' between the sphere and the interface. Thus, as noted earlier, a definitive conclusion was never possible as to the final mode of breakthrough. In some cases, however, the rates of change of the film or tail thickness do provide strong circumstantial evidence to support either film rupture or tail rupture as the likely mechanism of breakthrough.

In order to provide a framework for discussion of the results, it is useful to begin by reviewing the expected role of viscous, capillary and body forces in determining the degree of interface deformation for the case of sphere motion at a constant velocity. From a macroscopic point of view, a basic energy balance at any moment will exist between the rate of working, $F U$, by the particle on the fluid (where $U$ is the particle velocity and $F$ is the hydrodynamic drag which, at steady state, is equal in magnitude to the applied force necessary to maintain the constant velocity $U$ ), and the rate of conversion to internal and potential energy. When the particle is far from the interface, the rate of working, $F U$, is converted entirely to heat via viscous dissipation. As the particle approaches the interface, however, the force, $F$, required to maintain the velocity $U$ increases, partly as a consequence of an increase in the rate of viscous dissipation and partly to balance the increase in potential energy of the system as the interface begins to deform so that the heavier fluid is carried across the plane, $z=0$, of the undeformed interface and the surface area is increased. If we focus on the region above $z=0$ that is occupied by fluid 2, as illustrated and considered in detail for a tailing configuration in the Appendix, an approximate balance exists between the rate of working $F U$, the rate of increase of potential energy as the volume of fluid is increased by 'entrainment' into the tail, the rate of increase of surface energy as the interfacial area is increased, the rate of working by surface stresses at the exterior boundaries of the film plus tail and the rate of viscous dissipation in the film plus tail.

The details of interface deformation, can only be determined by solving the full problem outlined in the previous section, and we shall present results of this type very shortly. Some general features can be understood, however, on the basis of the approximate energy balance in the film plus tail that we have just outlined. Let us 
consider initially the case $\lambda=0$. Now, for any given $\lambda$, the rate at which the interface deforms for a given rate of input of mechanical energy, $F U$, is determined by the incremental increase of potential energy that is required due to the increase of surface area and the increased volume of heavy fluid above the $(z=0)$-plane. Since the potential energy increase is proportional to the surface tension and the density difference between the two fluids, and $C a$ and $C g$ measure the characteristic magnitude of viscous forces relative to capillary and buoyancy forces, it is evident in the constant-velocity case that a decrease in $C a$ and/or $C g$ will tend to reduce the rate of interface deformation. Thus, for a given position of the sphere, the interface will tend to be less deformed for smaller values of $\mathrm{Ca}$ or $\mathrm{Cg}$. Of course, some of the mechanical energy is converted irreversibly to heat even for $\lambda=0$, but as $\lambda$ increases both the rate of dissipation within the film plus tail and the rate of working by viscous stresses at the boundary of the tail-film region will increase. Consequently, for fixed $C a$ and $C g$ (roughly, fixed $F U$ ), we may expect that the rate of deformation will decrease with increase of $\lambda$ since an increased fraction of the input energy is being dissipated to heat. Thus, again, for a given instantaneous position of the sphere, the interface will be less deformed as $\lambda$ increases. An upper bound on the amount of fluid which the sphere can carry across the $(z=0)$-plane corresponds to the case when viscous effects are negligible and the interfacial free energy is zero $(\mathrm{Ca}=\infty)$. In this. case, the maximum volume of entrained fluid will be determined by a balance between the force applied to the fluid from the sphere, $F$, and the total buoyancy force on the fluid in the film plus tail region. We shall see shortly, from our detailed solutions, that this balance is very closely approached for $\lambda=0$ even when $C a=O(1)$. This is illustrative of the fact that interfacial tension acts primarily to moderate the rate of entrainment across the interface at any moment by affecting the shape of the interface in an attempt to minimize the area (or curvature), but does not itself control the total amount of fluid which can be carried across the plane by entrainment. Indeed, as the density difference becomes very small (i.e. $C g \rightarrow \infty$ ), the maximum degree of interface deformation will become very large for any level of interfacial tension (Berdan \& Leal 1982). The role of non-zero values for the viscosity ratio, $\lambda$, is similar to interfacial tension in the sense that the interface deformation (and rate of entrainment) is decreased at any instant, relative to its value for $\lambda=0$, but a non-zero viscosity ratio does not in itself control the total maximum volume of fluid which can be entrained across the $(z=0)$-plane, this still being determined by the balance between drag and the net body force on the film plus tail.

It is important to recognize that the constant-velocity problem considered in this section is special in that a tailing configuration of interface deformation must ultimately be achieved in all cases in the absence of hydrodynamic instabilities leading to rupture of the interface (the exception being $C a=C g=0$, corresponding to a solid wall). For very small (but non-zero) values of $C a$ and/or $C g$, the interface will remain virtually flat until the sphere begins to penetrate the plane $z=0$, and the minimum dimension of the film which then forms will be exceedingly small compared to the radius of the sphere. Furthermore, the force required to move the sphere at constant velocity will become exceedingly large. Nevertheless, a film and tail must ultimately form. Whether such an extremely thin film could actually be realized in the 'real' problem, where additional effects such as Van der Waals forces are present is doubtful, but that is a question which cannot be answered in the present context where only hydrodynamic, surface-tension and body forces are considered. In addition, as a practical matter, the present numerical scheme is not well-suited to cases involving extremely thin films (or tails) and we are thus forced (by the cost 
of obtaining a solution) to stop some calculations involving small values of $C a$ and/or $C g$ before the sphere moves a significant distance across the $(z=0)$-plane. In these cases, it is important to recognize that the inability to proceed further with the solution does not mean that a tailing configuration will not ultimately appear in the constant-velocity case (assuming of course that no hydrodynamic instability is encountered). It is only the inability of our present scheme to generate the solution with reasonable economics for small $C a$ and/or $C g$ which prevents our demonstrating the tailing configuration which must inevitably occur. The constant-velocity problem does not, therefore, contribute directly to our understanding of the conditions for existence of the film drainage or tailing configurations which can occur in the case of motion under the action of a constant force. It does provide the simplest forum for understanding, in detail, the physics controlling interface deformation and that is its most important role. In addition, however, the constant-velocity case provides an essential basis for high resolution experimental observations over a wide range of $C a$ and $C g$ as are necessary to expose the role in the coalescence process of effects such as non-Newtonian rheology or inertia which cannot be studied easily by a theoretical (numerical) analysis (Geller 1986).

Let us now consider the results obtained from the numerical calculations in detail for the case of sphere motion at a constant velocity. The first case treated is $\lambda=1$, $C a=C g=1$. We choose to begin with this case because the 'characteristic' measures of viscous, surface-tension and gravity forces are all of equal magnitude and thus these forces should be expected to have a roughly equivalent role in the behaviour of the sphere and interface. Profiles showing the interface shape at equal increments of time (or particle displacement) are plotted in figure 5. The most obvious feature of this solution is the long, slender tail which evolves behind the sphere and the rather considerable volume of fluid which is carried across the interface by the sphere. The film over the front portion of the sphere does thin slowly, but in none of the cases shown in the figure is it particularly thin compared to the sphere radius. It is also noteworthy that none of the interface shapes drawn in figure 5 exhibit any indication of hydrodynamic or capillary instability; although the spatial resolution of our solution algorithm is such that disturbances on the scale of either the tail diameter or minimum sphere-interface separation could be detected if they were present. It may be noted in this regard that the linear stability analysis of Lang \& Wilke (1971) leads to the conclusion that the 'film' in front of a rigid sphere approaching an interface should be stable in the absence of Van der Waals forces. On the other hand, the experimental observations of Maru et al. (1971) show the onset and growth of a varicose disturbance on the 'tail' which does cause it to break apart, but only after the tail is approximately 120 particle radii in length, far beyond the final configuration which is attainable at reasonable cost with the current method of solution. The obvious alternative would seem to be a detailed stability analysis for a draining, extending tail, such as that shown in figure 5. Unfortunately, none of the existing analyses of 'thread' stability is directly applicable, and an improved analysis does not appear viable to us at the present time due to the complicated and 'unknown' nature of the 'base' geometry and flow. The classical analyses of Rayleigh (1892) and Tomotika (1936) for surface-tension-driven instability of a stationary thread neglect the non-uniform geometry, the draining flow in the tail, and, most importantly the extension of the tail with time, which is known from both experimental and theoretical studies to increase stability, cf. Grace (1971), Chin \& Han (1979) and Olbricht \& Leal (1983). A problem which more closely resembles the situation in the tail is the stability of an infinite thread that is aligned with the symmetry axis of 


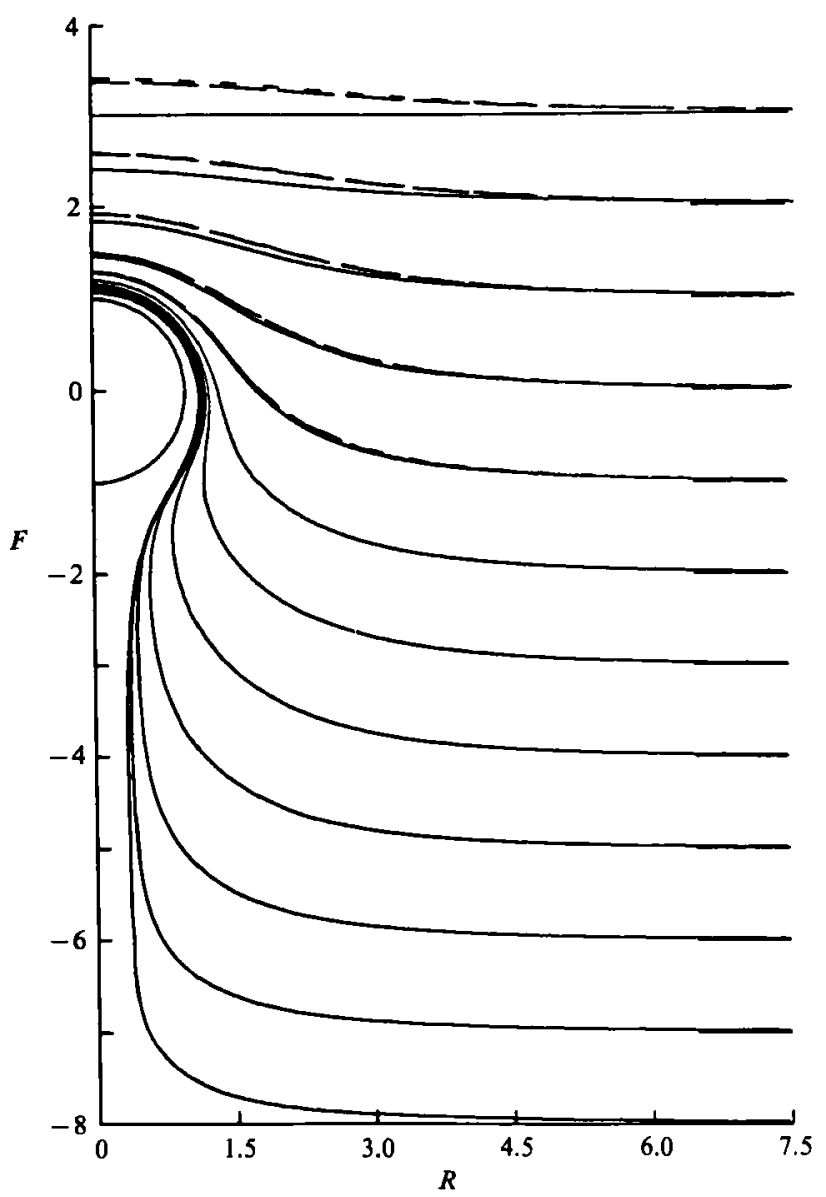

FigURe 5. Interface shape as a function of sphere position (drawn in a reference frame in which the sphere is fixed) for $\lambda=1, C a=1, C g=1$; - , shapes for sphere initially at $l_{0}=3$; ---, $l_{0}=5 ; \cdots, l_{0}=10$. Constant-velocity case.

a uniaxial straining flow, treated by Mikami, Cox \& Mason (1975). Their analysis includes the effect on stability of extension and of a decrease in thread diameter, but in a controlled manner determined by the assumed uniaxial straining flow (which is not present in the tail behind the sphere). These researchers predicted a fastest growing wavelength which depends on the instantaneous radius but which reaches an asymptotic limit with time. For the case $\lambda=1, C a=1$, this asymptotic limiting wavelength is approximately 35 thread radii (or roughly 15 sphere radii for our case); our calculations go as far as a length of about 6.5 for the tail region, considerably less than the wavelength for instability predicted by Mikami et al., and much less than seen experimentally by Maru et al. (1971). If, as said before, extension and thinning of the fluid cylinder are factors stabilizing the growth of capillary waves, Mikami's results may provide an upper bound on the length that the tail can achieve since the flow assumed in their calculations provides the greatest extensional character.

An examination of the governing equations and boundary conditions, (2)-(8), indicates that the interface shape will depend on the parameters $\lambda, C a$ and $C g$. Lee \& Leal (1982) have previously discussed the role of each of $\lambda, C a$ and $C g$ on the 


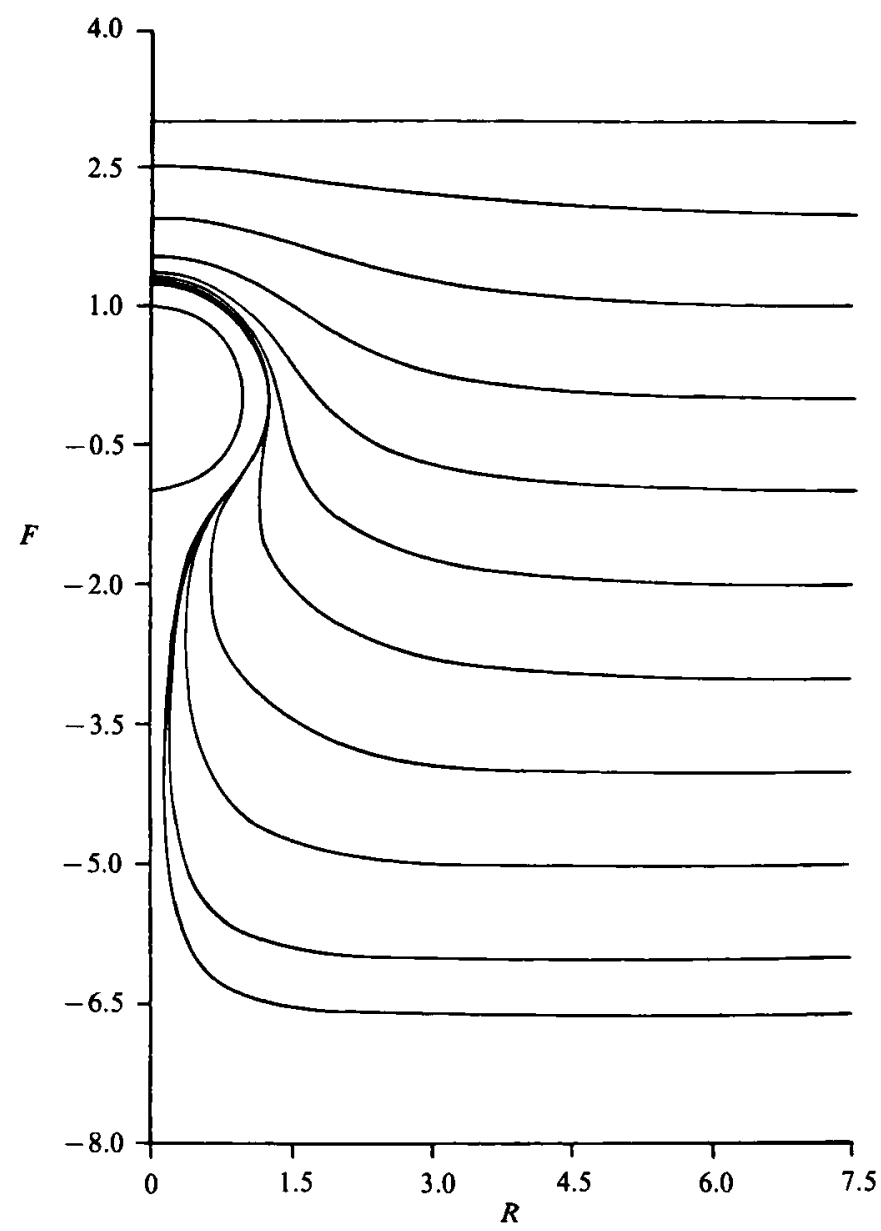

Figure 6. Interface shape as a function of sphere position for $\lambda=0, C a=1, C g=1, l_{0}=3$. Constant-velocity case.

behaviour of the deforming interface at small and moderate deformations. In the remainder of this section, we focus on the large deformation behaviour of the interface beginning with the influence of the viscosity ratio $\lambda$. The effect of the viscosity ratio can be seen by comparing the results in figure 5 with those in figures 6 and 7 , where we consider the same values of $C a=C g=1$, but $\lambda=0$ and 10 , respectively. It is visually evident on the basis of the film thickness at the front stagnation point of the sphere that the amount of deformation for a given position of the sphere is decreased in the early part of the deformation process as the viscosity ratio, $\lambda$ is increased. This visual impression is confirmed if we actually measure the volume of fluid in the film plus tail region above the $(z=0)$-plane as a function of particle position, though the differences on this basis between the cases $\lambda=0$ and $\lambda=1$ are very small. This can be seen in figure 8 where we have plotted (for reasons to be discussed shortly) the total volume of the fluid region plus sphere normalized by $6 \pi a^{2}$. We have argued earlier that the influence of increased viscosity in the upper fluid should be primarily one of controlling the rate and details of interface deformation rather than the maximum amount of fluid which can be carried across the $(z=0)$-plane. This conjecture is confirmed for $\lambda=0$ and 1 by the results in figure 8 , where a 


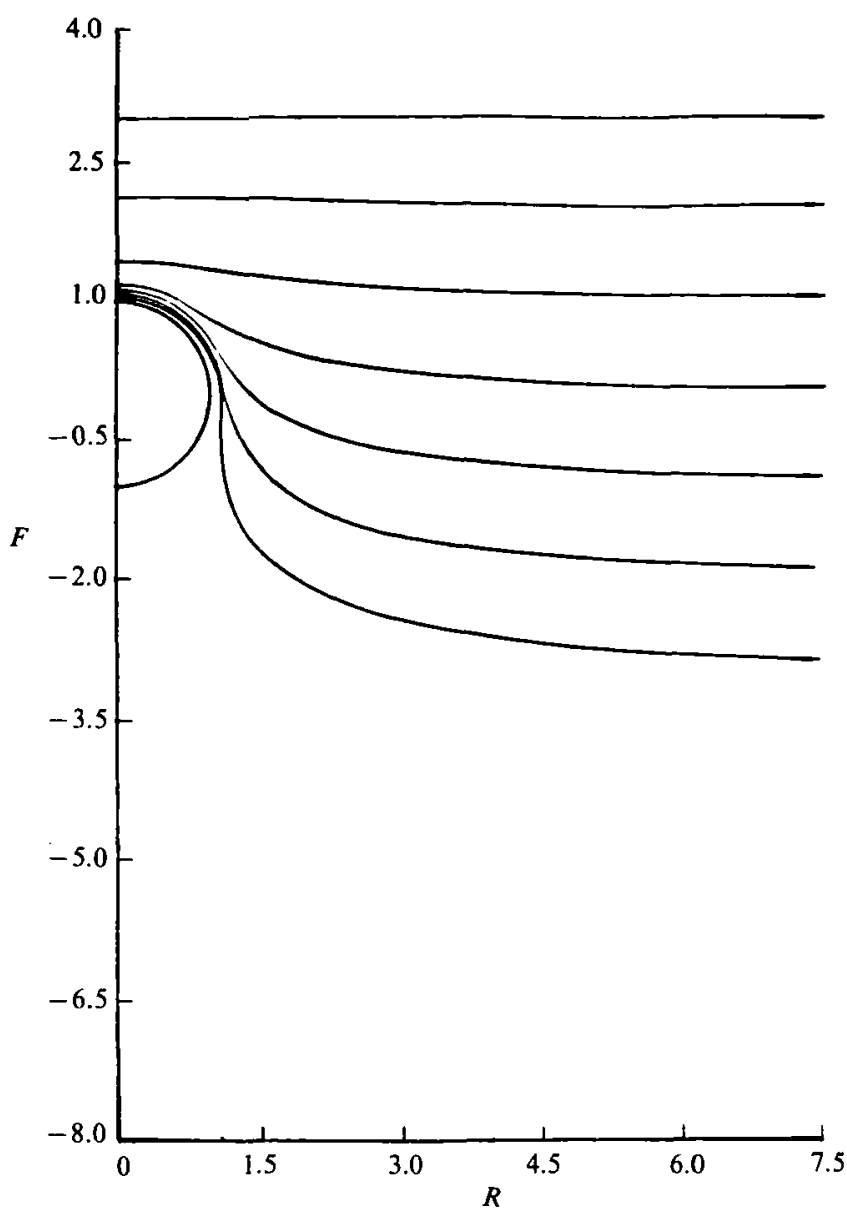

Figure 7. Interface shape as a function of sphere position for $\lambda=10, C a=1, C g=1, l_{0}=3$. Constant-velocity case.

maximum is obvious in the volume which is approximately independent of $\lambda$, though occurring somewhat later (i.e. for more negative values of $l$ ) for $\lambda=1$ than for $\lambda=0$. Beyond the point of maximum volume, the film plus tail region begins to lose fluid by drainage, though this process is also slower for $\lambda=1$ than for $\lambda=0$. The case $\lambda=10$, which initially begins with a much smaller volume of entrained fluid (for $l>0$ ) has not yet yielded a maximum in the volume of entrained fluid by the time that the calculation was stopped due to the thinness of the film at the front of the sphere, but the total volume of fluid entrained is similar to the other cases at intermediate values of $l$. In all of the general features described above, the numerical solutions illustrated in figures 5-7 confirm the qualitative physical picture (outlined at the beginning of this section) of the role of $\lambda$ in the interface deformation process. Certain detailed features of interface shape could not be predicted by the qualitative arguments, however, and these are also of interest here.

First, it is evident that the portion of the tail plus film region which changes shape most rapidly once the total volume begins to decrease by drainage is strongly dependent on $\lambda$. In particular, for $\lambda=0$, the film region barely thins at all but instead is carried along in almost rigid motion by the sphere. All of the loss of fluid volume occurs in the tail, which thins rapidly both because it is being stretched and because 


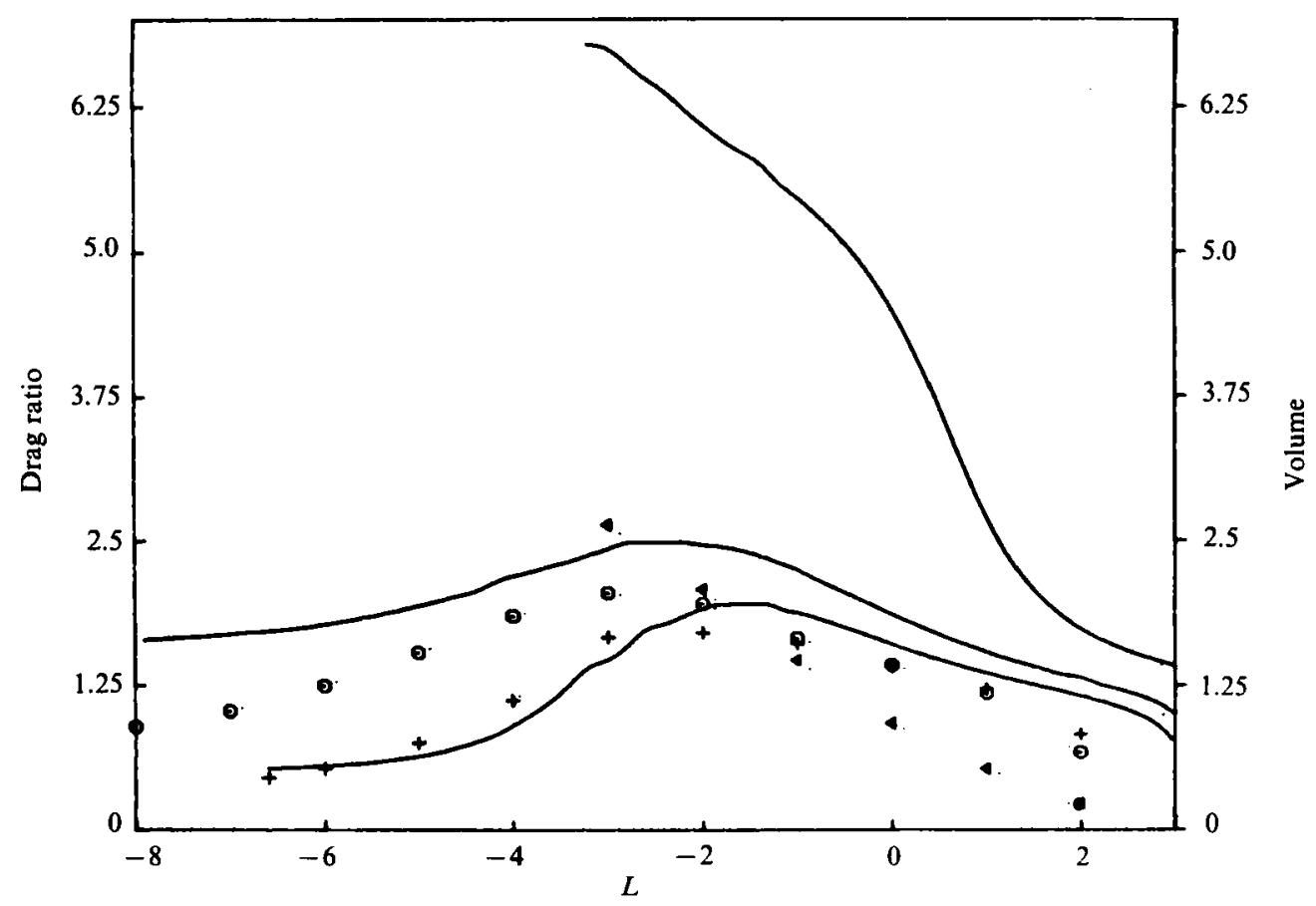

Fiqure 8. Drag ratio vs. sphere position for $C a=C g=1, \lambda=0,1$ and 10: - , numerically calculated values; volume of the tail plus sphere measured from the numerically calculated interface profiles divided by $6 \pi a^{3} ;+,(\lambda=0) ; \boldsymbol{0},(\lambda=1) ; \bullet,(\lambda=10)$.

there is no flow entering the tail from the film to compensate for this stretching and for the drainage of fluid back across the $(z=0)$-plane. For $\lambda=1$, on the other hand, the film is thinned more rapidly as a consequence of viscous stresses at the interface, the fluid in the tail drains more slowly and the result is a marked decrease in the rate at which the tail decreases in radius. The fact that the film barely thins at all when viscous stresses are removed from the interface (in setting $\lambda=0$ ) shows that neither capillary nor body forces play a significant role in this process, at least for $C a=C g=O(1)$. Finally, although we have indicated previously that the mode of breakthrough cannot generally be established in the absence of a thorough study of possible hydrodynamic instabilities, and/or other phenomena associated with the existence of colloidal forces between the interface and sphere surface, or between the interface and itself (for example, across the thin tail), a simple comparison of the rate of decrease of the film and tail thickness in the case $\lambda=0$ is strongly suggestive that breakthrough will occur in that case by a necking failure in the tail, leading presumably to a sphere in fluid 1 surrounded by a layer of fluid 2 (though this cannot represent an equilibrium configuration, see Johnson \& Sadhal 1985).

A final point of interest, with regard to these first three solutions, is the variation of the hydrodynamic drag on the particle as a function of its position. The numerically calculated values of the drag ratio (i.e. the drag/ $6 \pi \mu_{2} a U$ ) for the cases $\lambda=0,1$ and 10 with $C a=C g=1$, are shown as a function of particle position by the solid lines in figure 8. The deviation in the drag from Stokes' law obviously increases with $\lambda$. Furthermore, the results for $\lambda=0$ and $\lambda=1$ show a definite maximum in the drag at a position where the particle has passed beyond the plane of the undisturbed interface. This maximum in the drag appears to occur primarily because of a 
corresponding maximum in the volume of fluid in the complete column consisting of the film and tail. To demonstrate this fact, let us consider the net body force on the fluid column excluding the sphere which is simply $\left(\rho_{1}-\rho_{2}\right) g\left(v_{\mathrm{f}}+v_{\mathrm{s}}\right)$, where $v_{\mathrm{p}}$ is the total volume of fluid in the column (i.e. film plus tail), and $v_{\mathrm{s}}$ is the volume of the sphere. If the contributions of viscous stresses and interfacial tension were completely negligible in the macroscopic energy balance described earlier, this net body force should exactly balance the hydrodynamic force from the particle to the fluid. In this case,

$$
\text { Drag ratio }=\frac{v_{\mathrm{f}}+v_{\mathrm{s}}}{6 \pi a^{3}} C g^{-1}
$$

$$
\text { Thus, for } C g=1, \quad \text { Drag ratio }=\frac{v_{\mathrm{f}}+v_{\mathrm{s}}}{6 \pi a^{3}} \text {, }
$$

and it would follow that any variation in the drag ratio with particle position should be reflected in temporal variations of $v_{\mathrm{p}}+v_{\mathrm{s}}$. In order to test this conclusion, values of $v_{\mathrm{s}}+v_{\mathrm{f}}$ were measured from the numerical solutions, and $\left(v_{\mathrm{s}}+v_{\mathrm{f}}\right) / 6 \pi \boldsymbol{a}^{3}$ was plotted in figure 8 for comparison with the numerically calculated drag ratio. It is evident that the drag ratio and $\left(v_{\mathrm{f}}+v_{\mathrm{s}}\right) / 6 \pi a^{3}$ agree extremely well for the case $\lambda=0$. Apparently, any contributions of viscous dissipation or the increase in surface energy due to increased surface area to the overall energy balance (from which the simple force balance of (30) is derived) are negligible for $\lambda=0, C a=C g=1$. On the other hand, as $\lambda$ increases, the drag ratio considerably exceeds $\left(v_{\mathrm{s}}+v_{\mathrm{f}}\right) / 6 \pi a^{3}$, though the position of the maximum in the drag ratio still appears to agree well with the position of the maximum in $\left(v_{\mathrm{f}}+v_{\mathrm{s}}\right)$. We believe that the difference between the drag ratio and $\left(v_{\mathrm{s}}+v_{\mathrm{p}}\right) / 6 \pi a^{3}$ for $\lambda>0$ is primarily a consequence of the increased rate of working by viscous stresses at the interfacial boundary of the columnar region. The quantitative comparison for $\lambda=0$ provides strong evidence for the validity of the qualitative picture, based on an overall energy balance, that was outlined at the beginning of this section.

In the remainder of this section, we consider the detailed effects of variations in $C a$ and $C g$ on the large deformation behaviour of the interfaces. We first consider two cases of small $C a$ and $C g$. Figure 9 shows interface shapes for the case $\lambda=1$, $C a=C g=0.1$ and figure 10 presents the results for $\lambda=1, C a=C g=10^{-3}$ both with the centre of the sphere initially 3 radii away from a flat interface. The asymptotic limiting case, $C a \ll 1, C g \ll 1$, corresponds to very large surface tension and gravitational forces relative to viscous forces, and is known to yield asymptotically small deformations of the initially flat interface provided only that the sphere has not begun to penetrate the plane of the undisturbed interface. Although it is not known precisely how small $C a$ and $C g$ must be for this asymptotic behaviour to manifest itself, it might be supposed that simple dominance of surface tension or gravitational forces (corresponding to $C a$ or $C g<1$ ) would be enough. It is evident from figure 9 that this is not the case. Even for $C a=C g=0.1$, there is considerable deformation before the sphere comes close to the $(z=0)$-plane. The role of viscous forces is small in this case relative to surface tension and gravity effects so we see, (as was also true for $\lambda=0$ ), that there is only very slow thinning in the film ahead of the sphere in spite of the fact that $\lambda=1$. The important effects are taking place in the tail behind the sphere. Unlike the earlier results, a long tail is not formed behind the sphere. What is evident from figure 9 is that once the interface has been deformed by the sphere, say, between $l=-1$ and -2 , the relatively large surface tension tends to drive the interface towards a minimum energy configuration, namely a spherical shell encapsulating the 


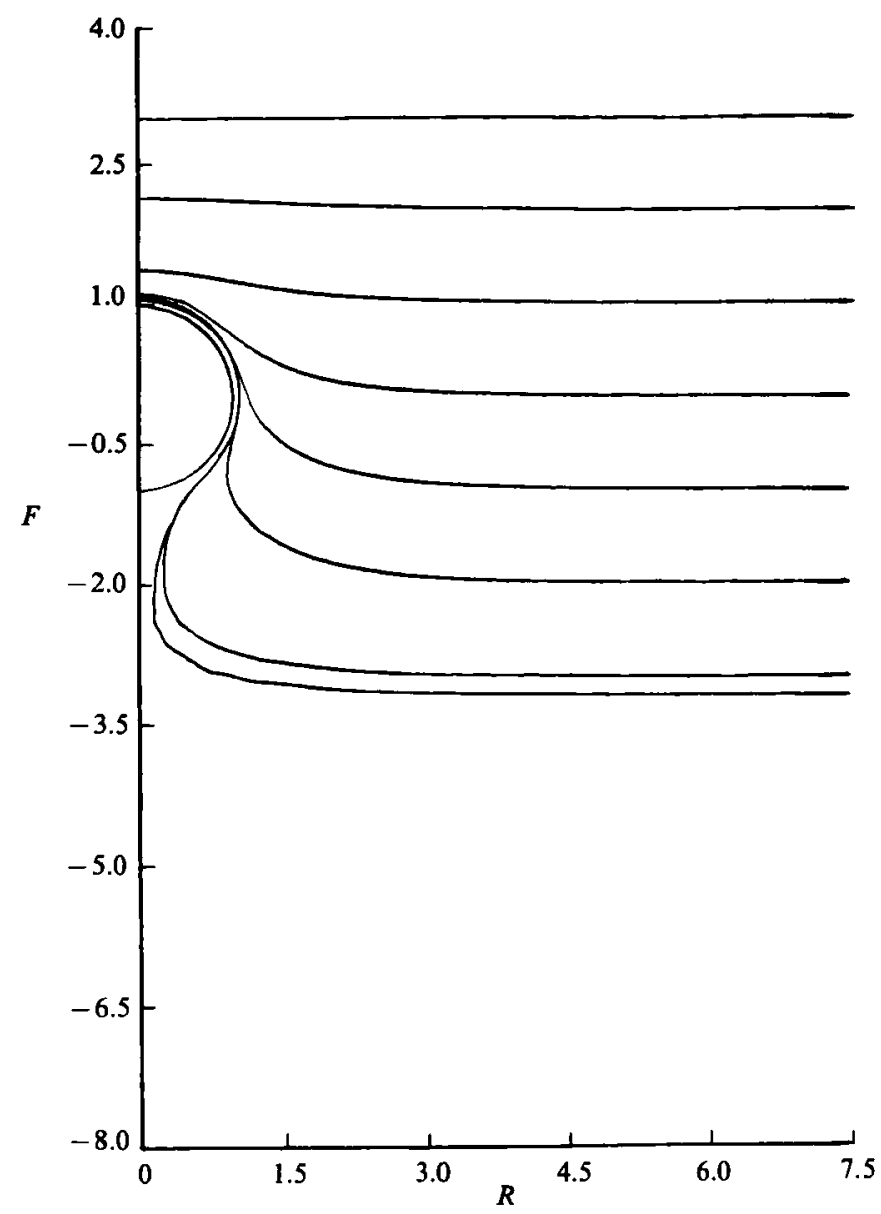

FIgure 9. Interface shape as a function of sphere position for $\lambda=1, C a=0.1, C g=0.1, l_{0}=3$. Constant-velocity case.

particle with a flat interface below. At the same time, the relatively strong capillary forces, in combination with the increased density difference between the two fluids, causes rapid drainage and a pinching off of the tail behind the sphere. In the second case, $\lambda=1, C a=C g=10^{-3}$, the magnitude of the surface tension and density difference is such that the interface appears nearly rigid at the point where the computation is terminated. Breakthrough, in this case, undoubtedly occurs in real systems by rupture of the thin film on the front portion of the body. Nevertheless, if we were to continue the present calculations for a sufficiently long time, a tail would eventually develop.

Figure 11 shows the drag ratio versus position for $\lambda=1, C a=C g=1,0.1$ and $10^{-3}$. The points which appear with the plot for $C a=C g=10^{-3}$ are exact analytical results for sphere motion towards a flat interface with $\lambda=1$ calculated by Lee \& Leal (1980). The present numerical results can be seen to agree very well with these analytical results. It should be noted that this comparison provides a fairly critical test of the accuracy of the numerical scheme because the numerical solution difficulties are most severe where sphere-interface separation becomes very small. The generalities discussed with regard to figure 8 (such as the existence of a maximum in the drag) 


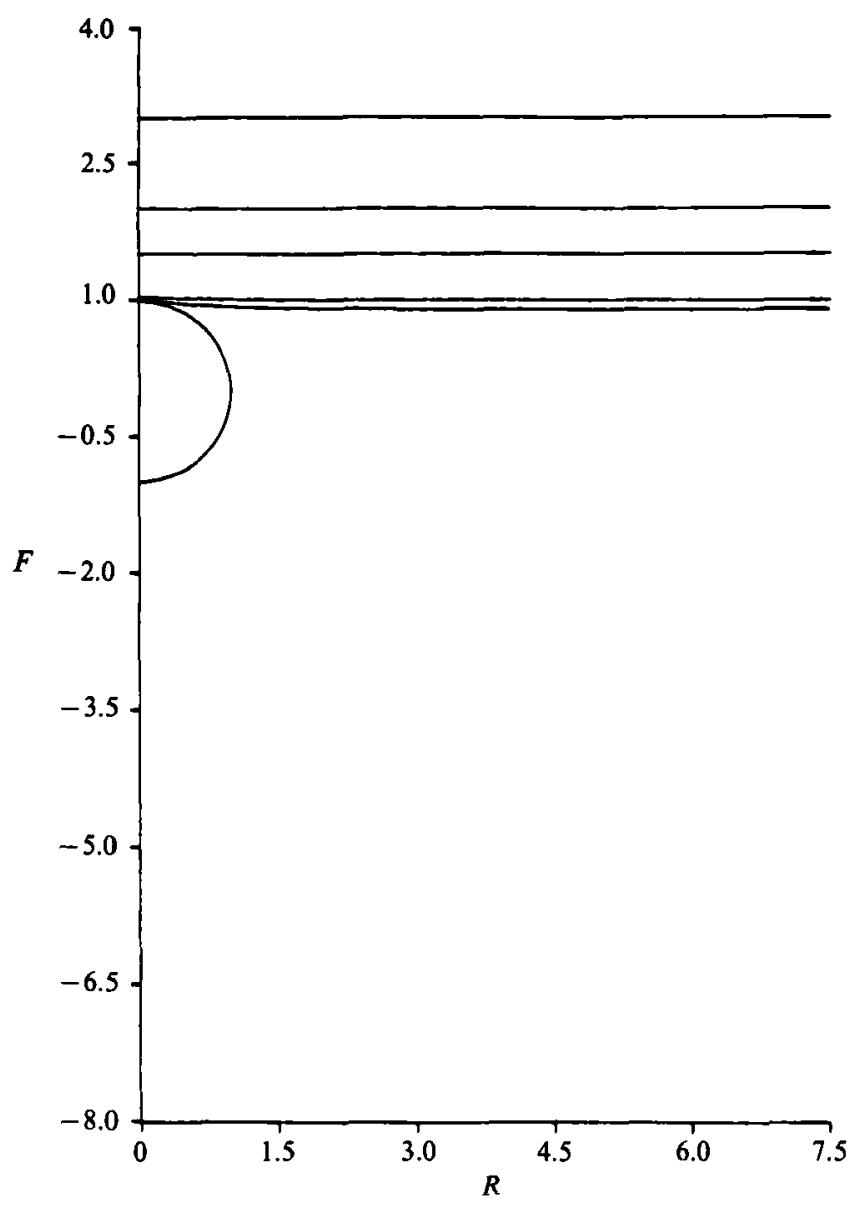

Figure 10. Interface shape as a function of sphere position for $\lambda=1, C a=10^{-3}, C g=10^{-3}$, $l_{0}=3$. Constant-velocity case.

are seen to apply in these cases as well; however, the increased restoring force associated with the decrease of $C a$ and $C g$ causes a very substantial increase in the magnitude of the drag ratio.

Finally, three constant velocity cases were considered in which $\lambda$ and $C g$ were held equal to 1.0, but $C a$ was varied to examine the effects of surface tension in more detail. In one of these cases, we took $C a=10^{-2}$, with the result that the interface deformed very little, and a deformation mode was observed similar to $C a=C g=10^{-3}$ but with slightly increased amplitude of deformation. In the interest of space conservation, this case is not shown here but will be available in Geller (1986). The second case, $\lambda=1, C g=1$ and $C a=\infty$ is depicted in figure 12. Here, the surface tension is zero. As the viscosity ratio is the main factor controlling the film thickness for either $\mathrm{Ca}$ or $C g=O(1)$, the thinning of fluid in front of the sphere is almost identical to that shown in figure 5 for $\lambda=C g=C a=1$. The difference in interface shapes between these two cases is in the thickness of the tail behind the sphere. In the present case without any surface tension, the tendency of the tail to pinch off is reduced, and a broader tail results.

The final case we present in the series illustrating the role of surface tension is 


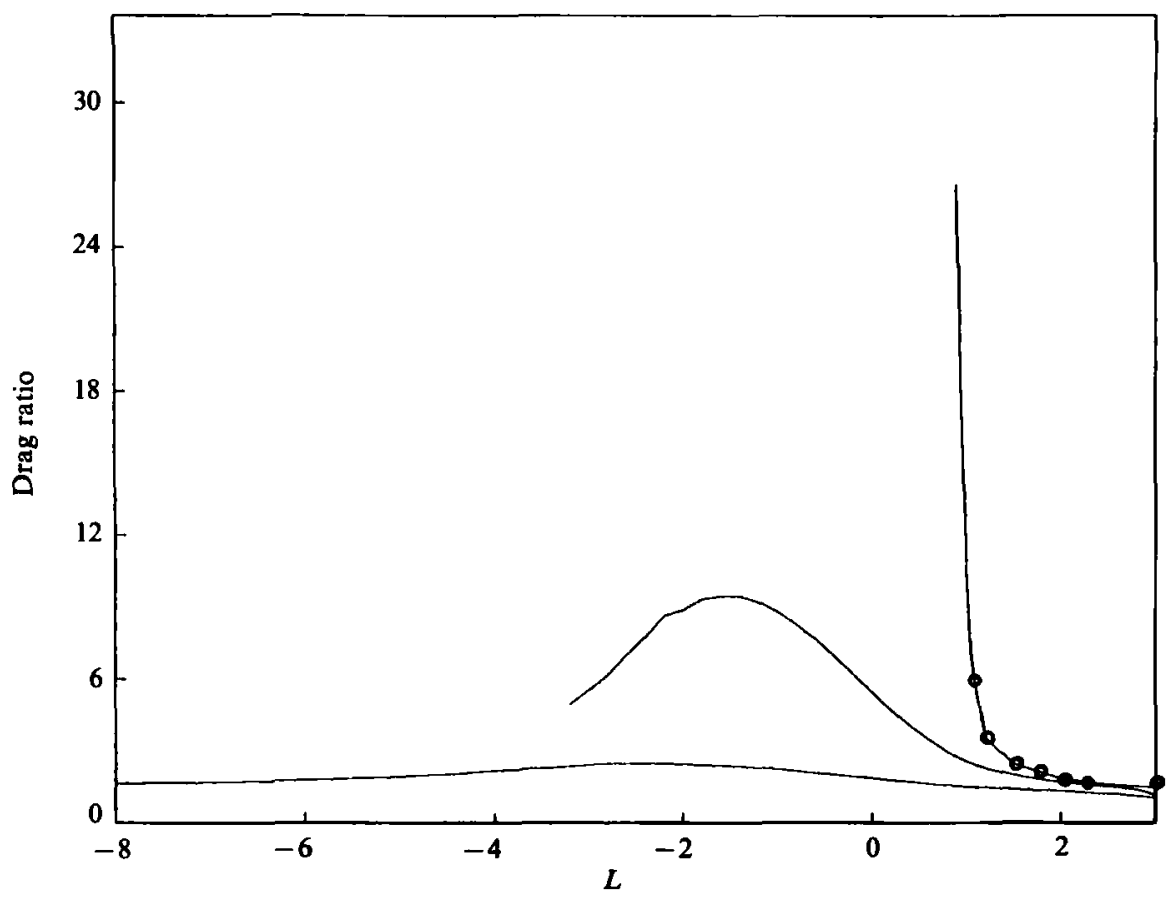

Figure 11. Drag ratio vs. sphere position for $C a=C g=1,0.1,10^{-3}, \lambda=1$, constant-velocity case. $O$, values calculated from Lee \& Leal (1980).

$C a=10^{-1}(\lambda=1, C g=1)$, shown in figure 13. In this case, $C a$ is small enough to have a very strong influence on the behaviour of the interface and yet not so small as to inhibit all deformation prior to the sphere crossing the $(z=0)$-plane. Examination of figure 13 shows that for the early stages of deformation, $l=3$ to 0 , surface tension acts to broaden the deformed part of the interface by flattening it, and thereby minimizing the total curvature. This broadening can be seen by comparison with the result for $C a=1$ in figure 5 . In spite of this broadening effect, however, the total volume of fluid carried across the plane of the initially undeformed interface is nearly equal for the two cases, consistent with the predictions from the macroscopic balance on the sphere-tail system. Furthermore, though the resistance of the interface to deformation caused by the large value of $\gamma$ initially results in a rapid thinning of the thin film in front of the particle, this film does not continue to drain as the sphere moves but eventually reaches a steady thickness equal to that for $C a=1, C g=1$, $\lambda=1$ as expected based on our earlier discussion of the role of the viscosity ratio in the formation of the film. As the sphere moves across the initial plane of the interface, $l=0$ to -3 , the interface must deform in front of the sphere, and the interface shape near the sphere is almost identical to the case (figure 5) where $C a=1$. However, the effect of surface tension is still to keep the interface as flat as possible, and the interface does not drop back to the $(z=0)$-plane as near to the centreline as for the $C a=1$ case. As a consequence, the volume of fluid entrained with the sphere for $C a=10^{-1}$ is somewhat larger during this period $(l=0$ to -3 ) than for $C a=1$ (as is the drag ratio). From $l=0$ to -3 , a transition is also beginning from one low-energy configuration to another. Through $l=-3$, surface tension acts to broaden the deformation in order to reduce the interfacial area. After this point, however, the deformation is great enough that the interface begins to move rapidly towards the 


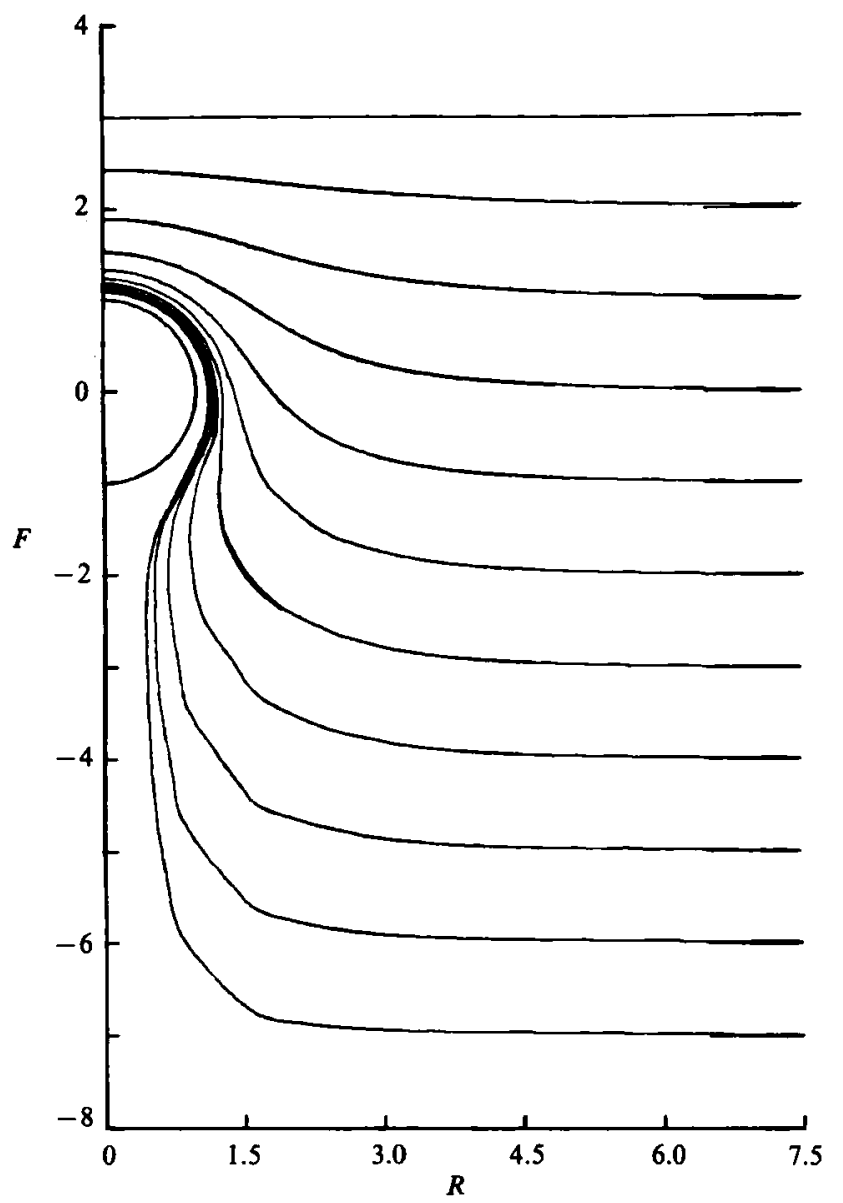

Figure 12. Interface shape as a function of sphere position for $\lambda=1, C a=\infty, C g=1, l_{0}=3$. Constant-velocity case.

other low-energy shape of an encapsulated sphere with the tail pinched off at the rear. Unlike the case presented earlier in which both $C a$ and $C g$ were $10^{-1}$, the primary mechanism driving the constriction in the present case is capillary forces, and it can be seen by comparison of figures 9 and 13 that the drainage occurs less rapidly. Nevertheless, drainage for $C a=10^{-1}, C g=1$ does occur faster than for the $C a=C g=1$ case, with $40 \%$ of the entrained volume being lost between $l=-4$ and -5 for the former case, and only $50 \%$ from $l=-4$ to -8 in the latter. The rapid change from one low-energy configuration to another causes a rapid pinching of the interface in the tail region so that the likely cause of breakthrough for cases like figures 9 and 13 with $\mathrm{Ca}=10^{-1}$ would seem to be 'pinch off' in which the thickness of the tail goes to zero, rather than the growth of a hydrodynamic instability in the tail as seems likely for $\lambda=1, C a=1, C g=1$.

The final constant-velocity case we present is for $\lambda=1, C a=1, C g=10^{-1}$. This is a case where the role of the density difference between the two fluids dominates over that of the viscosity ratio or surface tension. Figure 14 presents the interface shapes calculated for this system. The most noticeable feature of these shapes is that without surface tension to broaden the deformation, the interface falls back to the 


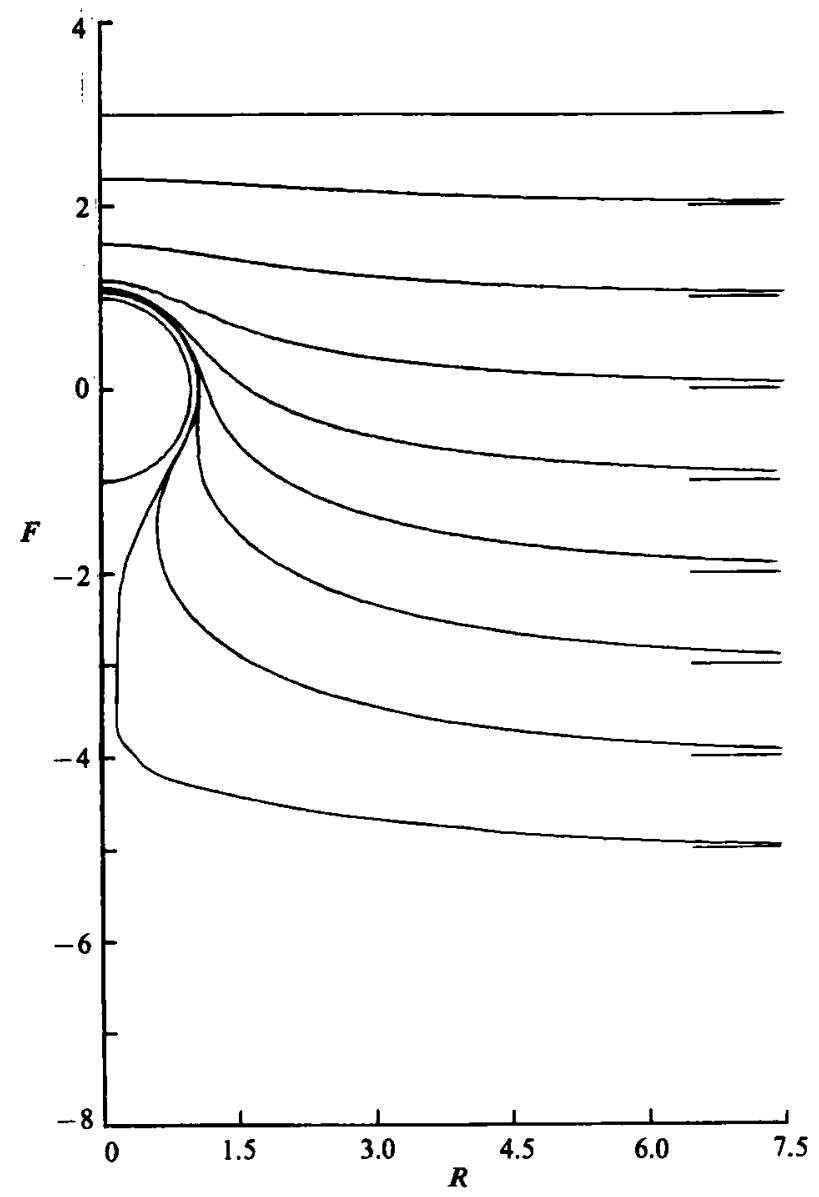

Figure 13. Interface shape as a function of sphere position for $\lambda=1, C a=0.1, C g=1, l_{0}=3$. Constant-velocity case.

$(z=0)$-plane relatively near the centreline. In most cases, the interface is flat beyond $r=1.5$. Also, although the magnitude of the drag ratio for this case is comparable to that for $\lambda=1, C a=10^{-1}, C g=1$ (figure 15); consistent with the results of the macroscopic balance (equation (30)), the volume of fluid carried across the $(z=0)$-plane is an order of magnitude smaller for the $C g=10^{-1}$ case. A noticeable difference in the drag ratio for the case $C g=10^{-1}, C a=1$ relative to the other two cases shown in figure 15 is that the drag ratio appears to be levelling off after $l=-3$, while the drag continues to decrease for the other two cases. This difference is attributable to the different mechanisms causing fluid to drain from the region behind the sphere. In the case where the density difference between the two fluids is the dominant driving force for drainage, the particle is almost entirely encapsulated and this combined body of a sphere and fluid film is moving as a single unit. This conclusion is supported not only by the interface shapes of figure 14, but also by the $u_{z}$ and $u_{r}$ values calculated for the interface in the encapsulated region. Behind the sphere, the fluid column is apparently no longer being supported by the viscous forces and so does not contribute to the drag on the sphere. In this case, $C_{\mathrm{D}}$ levels off near the value for an isolated sphere-film combination moving through fluid 1 . In the cases where surface tension is the major cause of drainage (figure 13), or is equal in 


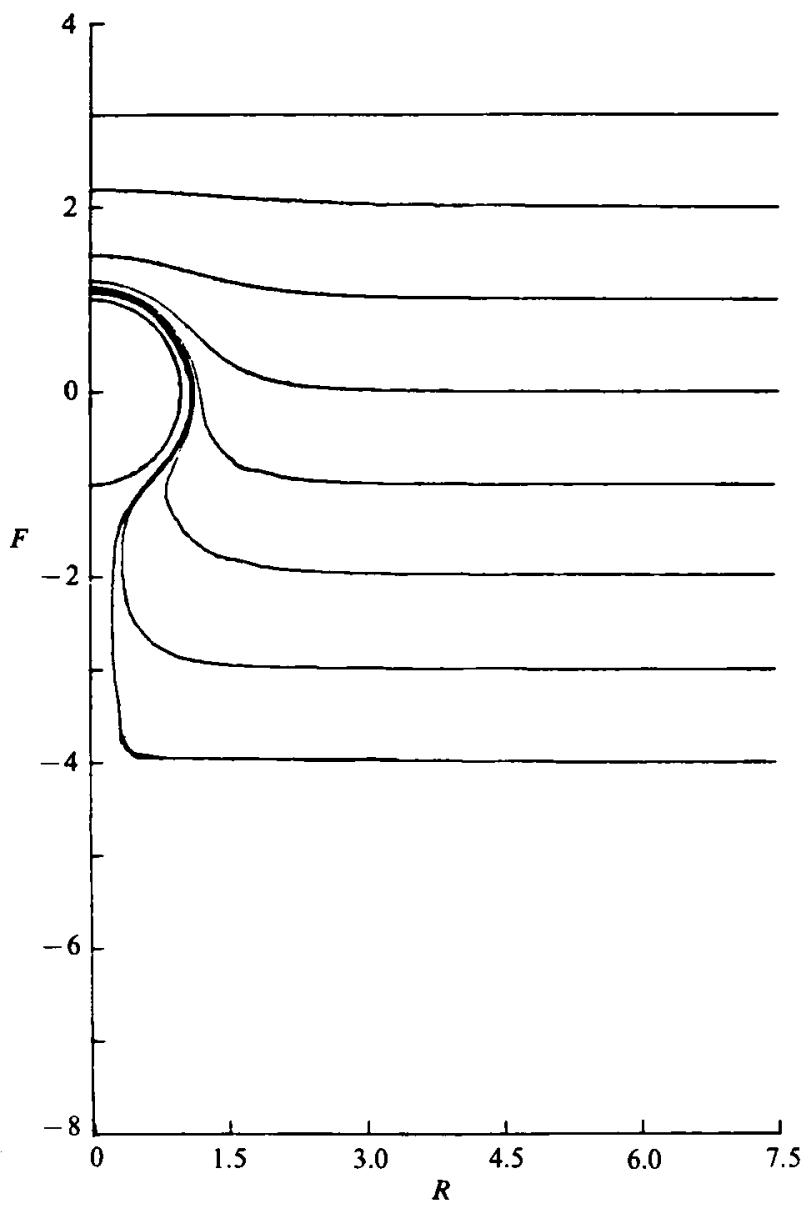

Fiqure 14. Interface shape as a function of sphere position for $\lambda=1, C a=1, C g=0.1, l_{0}=3$. Constant-velocity case.

importance to the density difference (figure 9), a significant force is being exerted on fluid 2 at the interface. In the film region in front of the sphere, the force due to surface tension acts in the direction opposite to sphere motion as the inward normal has a downward pointing $z$-component. After the sphere begins to become encapsulated, part of the interface near the sphere has an inward pointing normal whose $z$-component is in the same direction as the sphere's motion, and in this region the $z$-component of the normal force due to interfacial tension will be directed along the line of sphere motion thereby lowering the drag. As the area of the interface with upward pointing $z$-component of $n$ is growing (unlike the film region which has a downward pointing component of the normal but is stable in size), the net effect is for the drag on the sphere resulting from interfacial tension to decrease with time as the pinching continues. Since the pinching process will not stop until the sphere is entirely encapsulated and, as shown above, the drag following from the density difference levels off, the drag ratio continues to decrease for these two cases.

The results of this section provide definite evidence of a tailing configuration as the sphere passes across the plane of the undisturbed interface. The dynamics of the layer of fluid immediately adjacent to the sphere appears to be dominated by viscous forces, with gravitational forces also playing a role, but to be only weakly influenced 


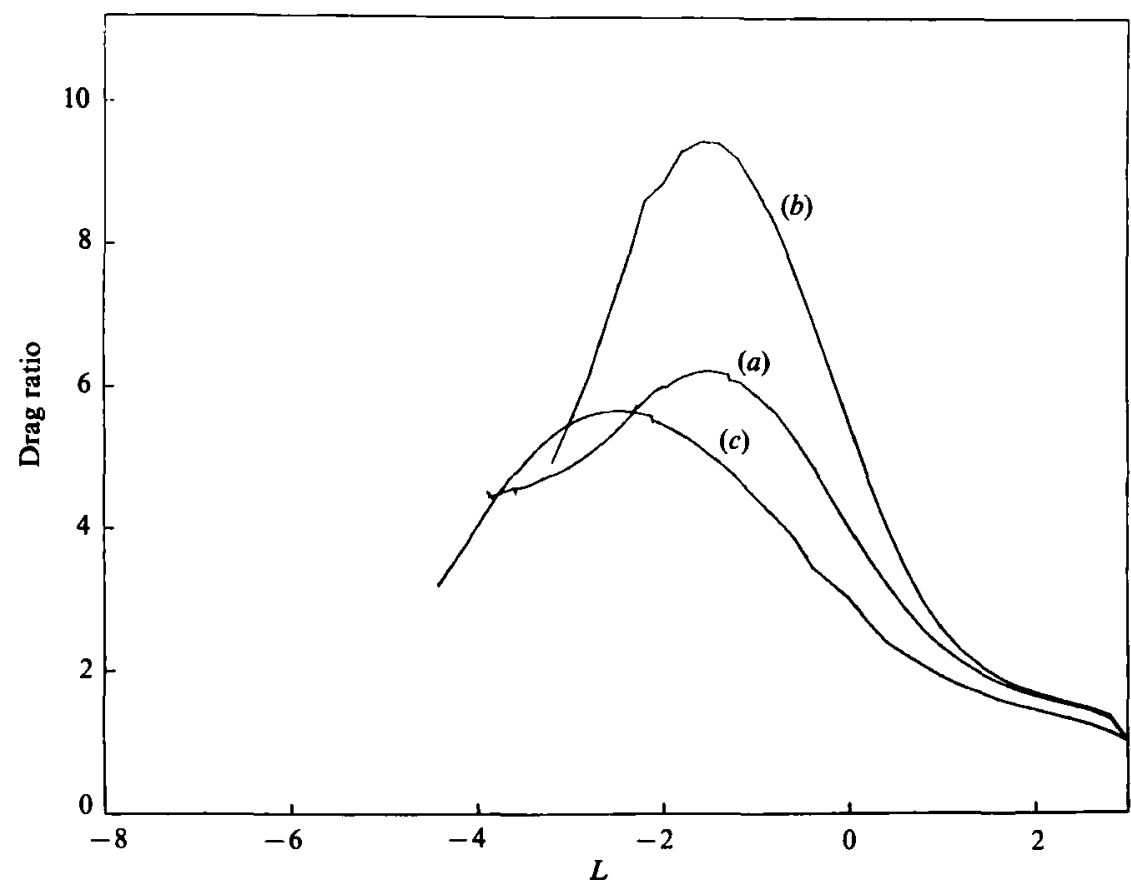

FIGURE 15. Drag ratio v8. sphere position for $\lambda=1:(a) C a=1, C g=0.1 ;(b) C a=0.1, C g=1$; (c) $C a=C g=0.1$.

by interfacial tension forces. The dynamics of the tail, on the other hand, appear to be more strongly influenced by interfacial tension forces, these tending to pinch off the tail as the system is eventually driven towards a second minimum free energy configuration in which the sphere is surrounded by a thin fluid layer of constant thickness and the interface reverts to a flat, undeformed configuration. In the next two sections we explore the effects of motion under the action of a constant force, rather than motion at constant velocity as in this section, and also consider the influence of the initial configuration for values $l_{0}<3$.

\section{Results for motion from a large distance due to a constant body force on the sphere}

In this section, we describe our results for the case of motion under the action of a constant external body force. Our goals are twofold. First, we wish to determine whether the conclusions of the preceding section, relating to the roles of $\lambda, C a$ and $\mathrm{Cg}$ in controlling the shape of the interface, carry over from the constant-velocity to the constant-force problem. Also, we are concerned with the conditions for the establishment of the tailing and film-drainage configurations. Unlike the constant velocity situation discussed in the previous section, where a tail will necessarily develop provided a large enough force is exerted on the sphere, in the constant-force case, either a tailing mode or film-drainage behaviour might result. The constant-force problem is fundamentally different from the earlier case in that here the velocity decreases as the sphere approaches the interface, hence $C a$ and $C g$ decrease effectively as well. It is possible that the particle force is not great enough at these lower velocities to carry it across the plane $z=0$ and form a tail, with the result that film 
draining is the exhibited mode of breakthrough. Finally, we wish to compare the results of our numerical solutions against those of earlier theories and experiments. To accomplish the latter goal, two calculations were carried out corresponding to published experimental runs. One was for a case which experimentally exhibited film drainage, the other where a tail configuration was reported.

Before proceeding to discuss numerical results, however, we note that it can be shown without use of a numerical procedure that only one mode of deformation is possible for a certain subclass of the constant-force problem. In particular, in cases where the force responsible for sphere motion is buoyancy-induced, film-drainage is the only possible configuration when the particle density is intermediate between the densities of the two fluids. This result is demonstrated easily by showing that any configuration in which the particle passes beyond the plane of the initial flat interface, is inconsistent with a macroscopic 'equilibrium' force balance when the sphere density is between that of the two fluids. The details of this calculation are shown in the Appendix. The condition of an intermediate sphere density is satisfied whenever $C g<\frac{2}{9}$. The fact that no inconsistency arises for sphere densities which are not intermediate to the two fluids (i.e. for $C g>\frac{2}{9}$ ) does not, of course, prove that a tailing configuration will actually arise in a dynamical calculation (or experiment). It shows only that such a configuration is possible.

We consider one case for which the macroscopic balance predicts film drainage, i.e. $C g<\frac{2}{9}$. This is for the set of parameters $\lambda=0.022, C a=0.464, C g=0.089$. These parameters correspond to an aluminium sphere falling through golden syrup toward liquid paraffin, the system used in Hartland's (1969) experiments. The associated Reynolds number for this system is 0.003 . A feature predicted in the film-drainage theories for this case is the pinch point described by Hartland (1969) and given a physical explanation by Jones \& Wilson (1978). Calculated interface shapes for the sphere starting at $l_{0}=3.0$ are shown in figure 16 . The mode of interface deformation is clearly film drainage. Figure 17 is an enlargement of the film region showing the pinching of the interface as predicted by Jones \& Wilson (1978). Also shown are the results from Hartland's (1969) experiments. Although the calculated shapes are qualitatively close to the measured interface positions, the quantitative agreement is not particularly good. We feel that this is most likely a manifestation of different initial configurations in the two cases. The initial condition for the numerical work has already been discussed. The sphere is placed with its centre 3 radii away from a flat interface and released subject to the condition of a constant applied force. Hartland is less clear about his initial conditions. In fact, the only evidence given is the interface shape for $t=1.89$. For the case $l_{0}=3$, calculated numerically, it takes a time of $5.50 \mathrm{~s}$ to reach a film thickness at the centre-line corresponding to this measured interface shape. From this, we conclude that the experimental run by Hartland started with the sphere closer to the interface than $l_{0}=3$, but we cannot be sure exactly where the experiment did start.

We now consider the more interesting cases $\left(C g>\frac{2}{9}\right)$ where a tailing configuration is possible and address the question of when tails actually occur. For the case of the sphere moving with constant velocity, $U$, the parameters $C a$ and $C g$ are defined in terms of $U$. In the present situation, however, the velocity of the sphere does not have a fixed value but changes with position. An appropriate characteristic velocity scale for dimensional analysis (cf. (2)-(8)) is the Stokes velocity for motion of the sphere under the same force in an unbounded fluid (i.e. sufficiently far from the interface). Values of $C a$ and $C g$ defined in terms of this velocity are then used to compare results with the constant-velocity case for the same values of $C a$ and $C g$. 


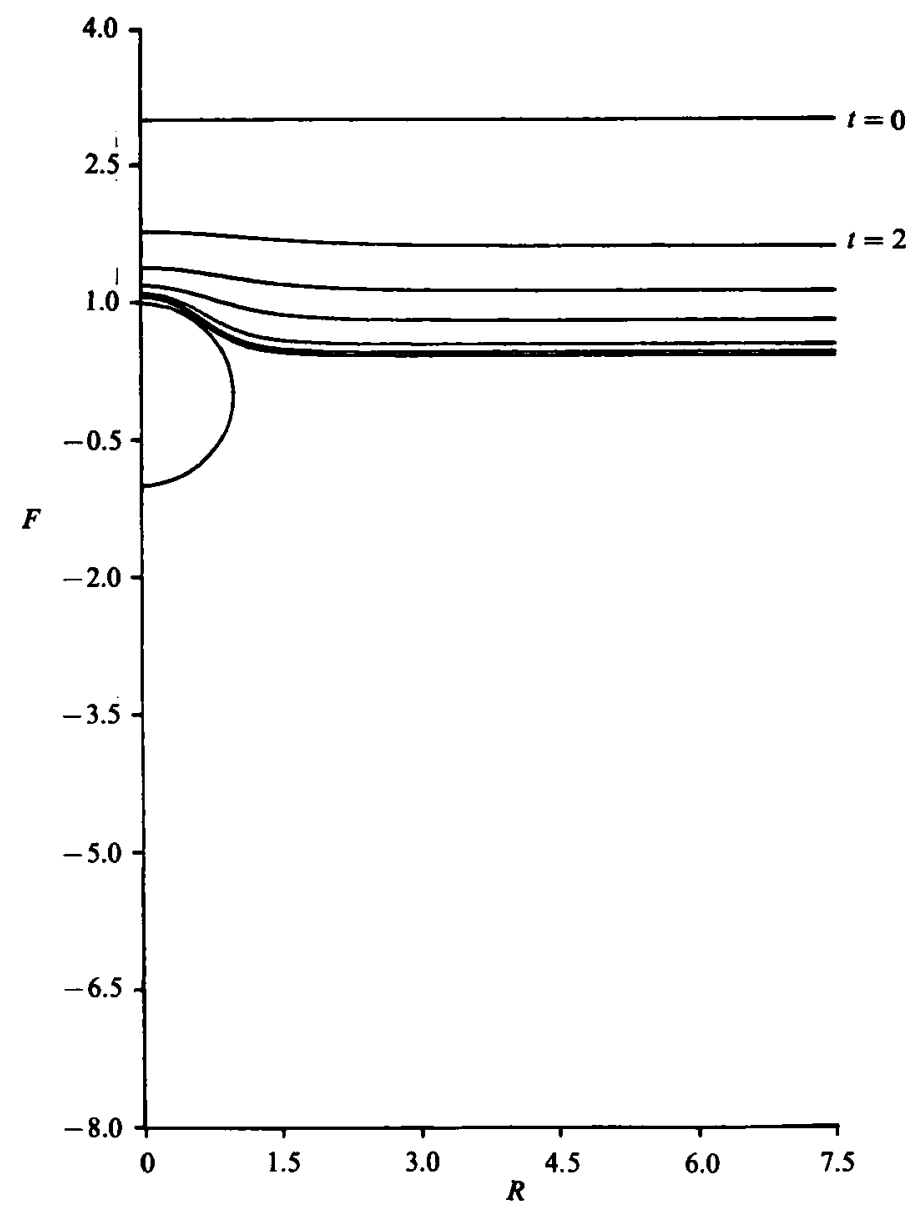

Figure 16. Interface shape as a function of sphere position for $\lambda=0.022, C a=0.465$, $C g=0.089, l_{0}=3$. Constant-force case. Interface shown for $t=0,2,3,4,6,8,9.5$.

However, the actual instantaneous sphere velocity varies with position, as already noted, and is generally less than the Stokes velocity. Thus, the values of $\mathrm{Ca}$ and $\mathrm{Cg}$ defined in terms of the Stokes velocity overestimate the magnitude of viscous forces relative to capillary or gravitational (buoyancy) forces for any given instantaneous position of the sphere. For some purposes, it is therefore useful to consider 'effective' values of $C a$ and $C g$ (denoted as $C a_{\text {eff }}$ and $C g_{\text {eff }}$ ) based on the instantaneous sphere velocity. The differences between characteristic values of $C a$ and $C g$, and their effective or instantaneous values, $C a_{\text {eff }}$ and $C g_{\text {eff }}$, are important in understanding the difference between motion at a constant velocity and motion with a constant applied force. Figure 18 shows the interface shape for $\lambda=1, C a=C g=1$ and $l_{0}=3$ with a constant applied force on the sphere, while the instantaneous sphere velocity is plotted in figure 19. It is evident that the sphere velocity is strongly decreased in the presence of the interface when moving under the action of a constant force. Thus, the deformation at any point in time is less than in the corresponding constant velocity case, as may be seen by comparing figures 5 and 18 .

However, there is no question that the deformation process (and thus, presumably, time to breakthrough) will be much longer for a given value of $C a$ and $C g$ in the 


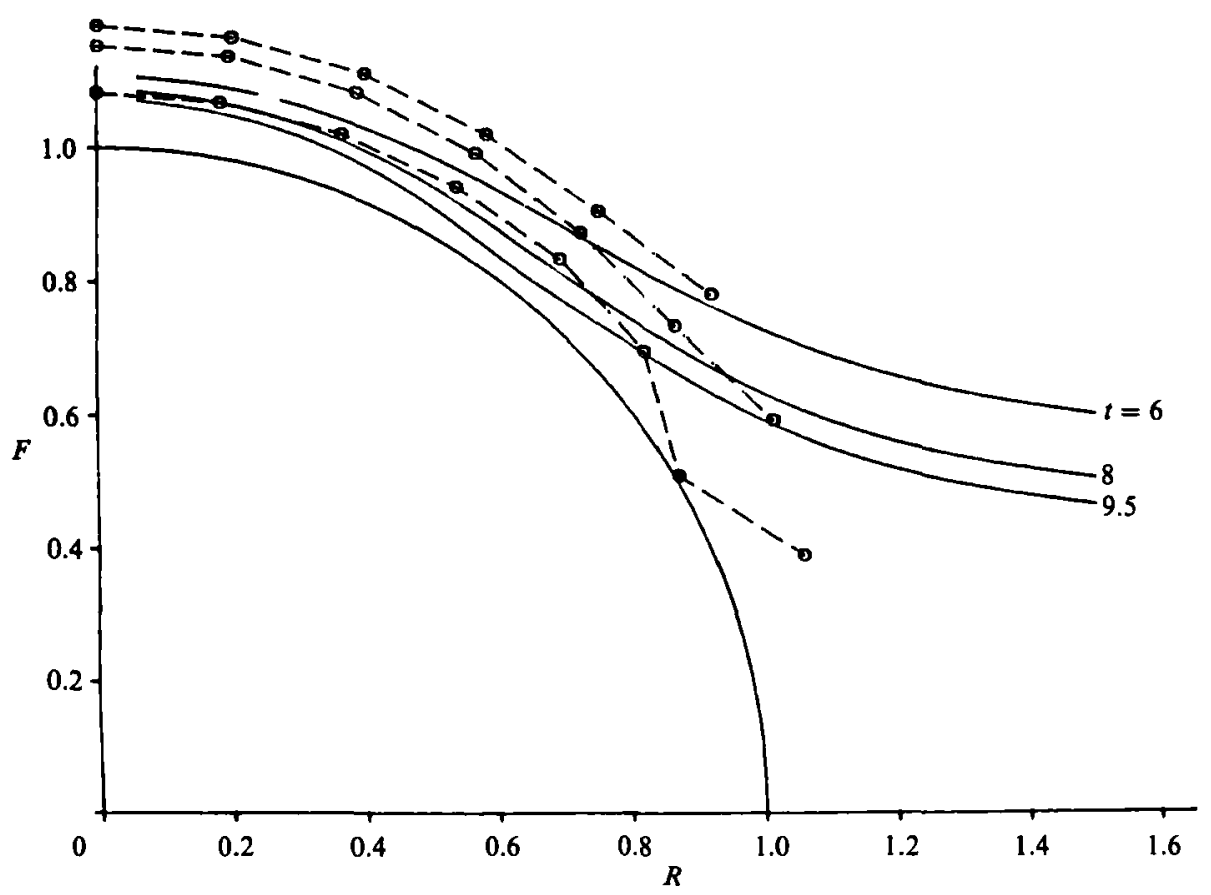

Figure 17. Detail of film region for $\lambda=0.022, C a=0.465, C g=0.089 ; l_{0}=3:-$, numerical calculations; -...-., experimental results from Hartland (1969).

constant-force problem. What is in doubt is the mode of deformation and ultimately breakthrough. For the purpose of answering this question and providing a more meaningful comparison of the constant-force and constant-velocity cases, interface shapes should be compared at the same positions of the sphere relative to the plane of the undeformed interface rather than at equal increments of time. Furthermore, based on the reduction in sphere velocity shown in figure 19 , the instantaneous or effective values of $C a$ and $C g$ in the case $\lambda=1, C a=1, C g=1$ range between initial values of $C a=C a_{\text {eff }}, C g=C g_{\text {eff }}=1$, and minimum values $C a_{\text {eff }}=C g_{\text {eff }}=0.09$ at $t \approx 15$. Thus, one might expect to find interface shapes which lie somewhere between the constant-velocity cases $\lambda=1, C a=1, C g=1$ and $\lambda=1, C a=10^{-1}, C g=10^{-1}$ that were shown in figures 5 and 9 , respectively. Comparison of figures 5, 9 and 18 shows that this is, indeed, the case. Compared with the interface shape at the same sphere position for the constant velocity problem with $C a=C g=1$, it is evident that the film is initially thinner in the constant-force solution, but that there is a stronger tendency later in the deformation process for the short tail behind the sphere to pinch off to produce a film of constant thickness over the majority of the sphere surface. Although this latter behaviour is strongly reminiscent of the constant-velocity case with $C a=C g=0.1$ (figure 9), the film thickness is considerably larger in the constant-force problem reflecting the relatively small forces resisting deformation at earlier times in the process when $C a_{\text {eff }}$ and $C g_{\text {eff }}$ are larger than 0.1. Although calculations for $t>25$ were not carried out, it appears very likely on the basis of comparison with the constant-velocity problem that the mode of breakthrough will be pinching off of the fluid tail behind the sphere.

Additional evidence for the existence of tailing modes of interface deformation in the case of sphere motion with a constant applied force will be presented shortly. It 


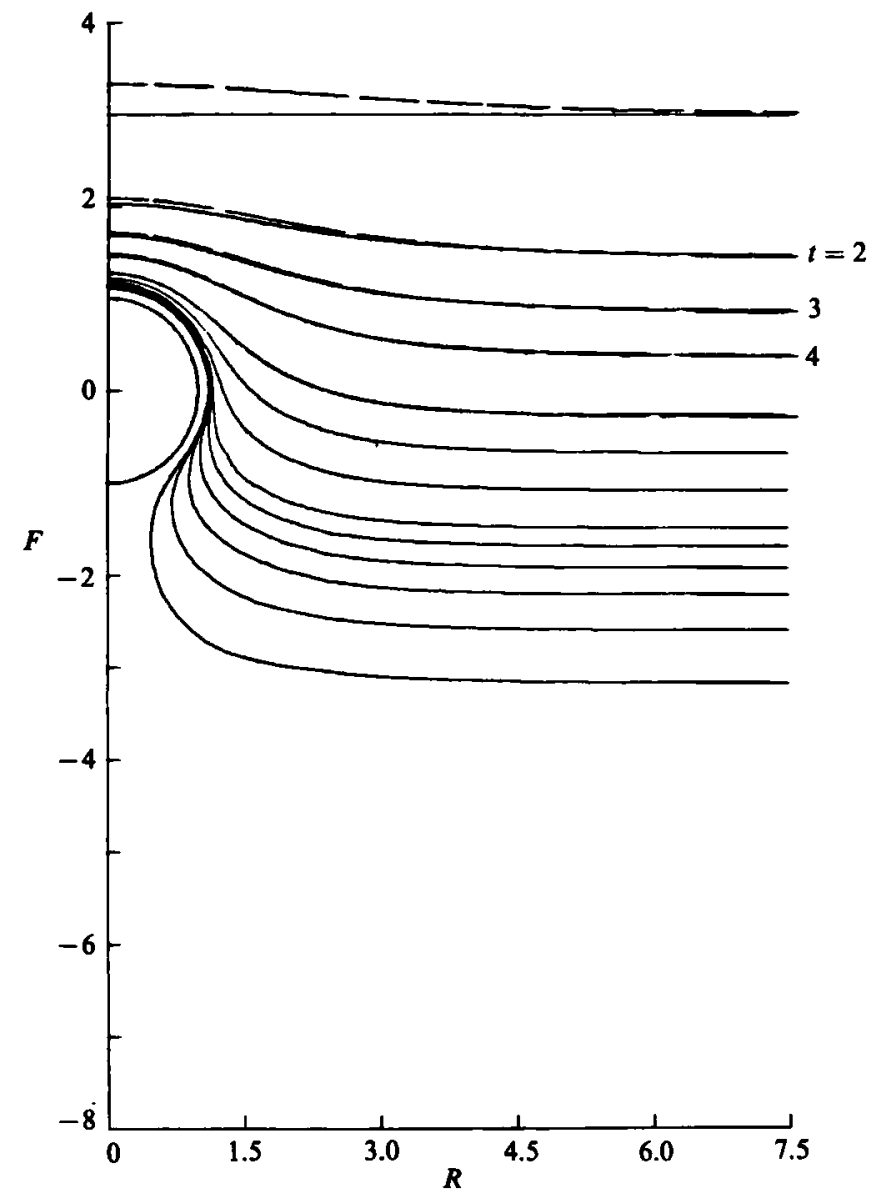

Figure 18. Interface shape as a function of sphere position for $\lambda=1, C a=1, C g=1$ : for sphere initially at $l_{0}=3 ;---, 5 ; \cdots,-, l_{0}=10$. Constant-force problem. Interface shown for $t=0,2,4,6,8,11,15,17,19,21,23,25$.

is apparent, however, that the rapid decrease in $C a_{\text {efr }}$ and $C g_{\text {eff }}$ which occurs as the sphere slows down does lead to much shorter tails for given values of $\mathrm{Ca}$ and $\mathrm{Cg}$ than occurred in the constant-velocity problem. The possibility exists that a given set of parameters which gave a high drag ratio and a broad tail behind the sphere for constant velocity could yield a film-drainage configuration in the constant-force case even for $C g>\frac{2}{9}$.

Figure 20 shows the interface configuration for the constant-force problem with $C a=C g=1, \lambda=10$, a case which did give a broad tailing configuration in the constant-velocity case. The sphere velocity is plotted against time in figure 21 . Comparing figures 20 and 7 one sees that the broad tail of the constant-velocity plot has become a film-drainage configuration in the constant-force problem. At $t=33$, the sphere velocity has dropped to 0.04 of the corresponding Stokes velocity, and thus $C a_{\text {eff }}=C g_{\text {eff }}=0.04$. With such large restoring forces relative to the viscous forces, further deformation is difficult so one expects the remaining fluid to merely drain away between the sphere and the interface.

Maru et al. (1971) have previously attempted to develop an a priori criteria for existence of a tailing configuration. The existence of serious shortcomings in this work has been mentioned in an earlier section. Here, comparison of present results for the 


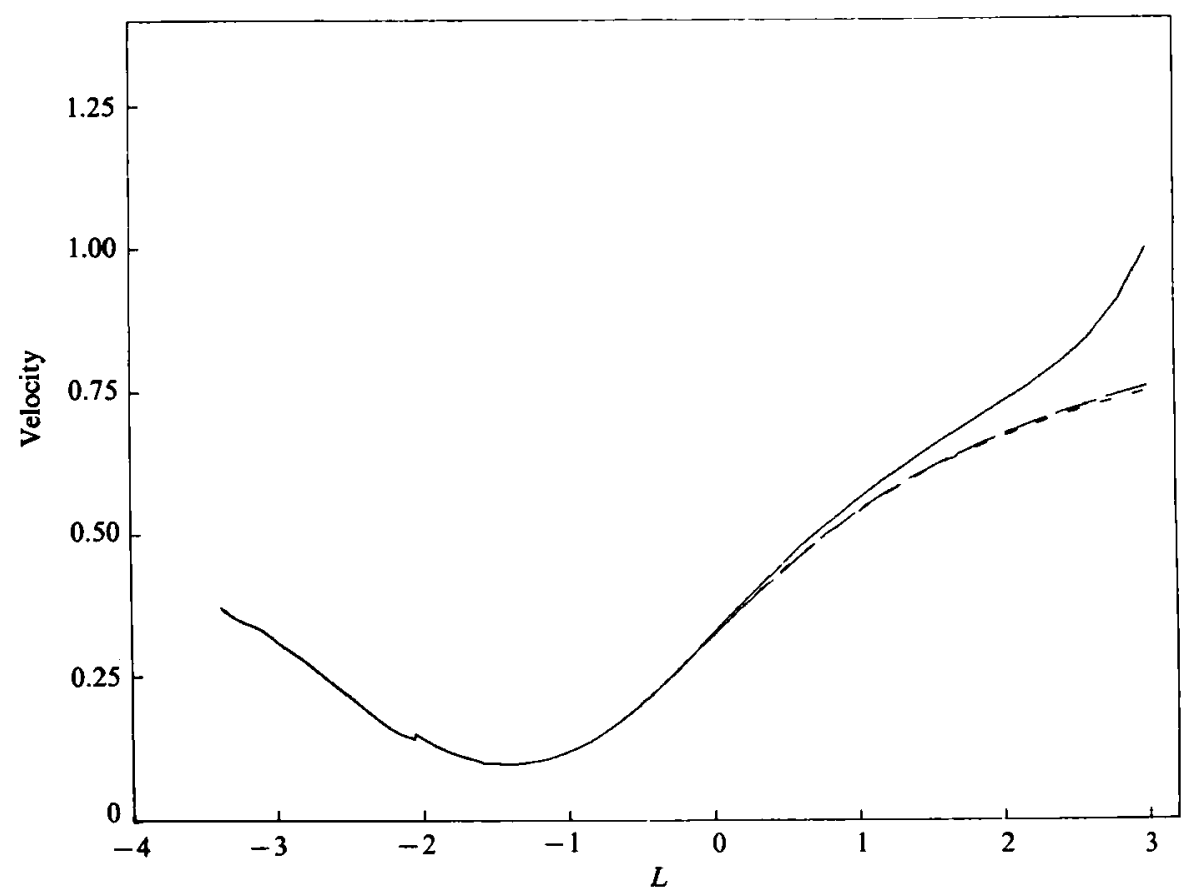

FIaure 19. Velocity as a function of sphere position for $\lambda=1, C a=1, C g=1:-, l_{0}=3$; ,$-- 5 ;-\cdots, l_{0}=10$.

various values of $\lambda$ points to one of the problems with their work and that of all others who use a quasi-static force balance to calculate sphere and interface motion. By failing to consider the shear stress on the surface of the interface, these authors do not have any way of judging the timescale of sphere motion relative to drainage of the film, and so cannot differentiate between systems with different viscosity ratios. In effect, they assume a film-drainage configuration as the initial condition and neglect the interface beyond the point where it begins to break away from the sphere. Figure 18 shows that the film region is established early and remains essentially the same after $t=8$ for $l_{0}=3$; whereas, the interface in the break-away region continues to deform creating a tail configuration. For the large-viscosity-ratio case and the same values of $C a$ and $C g$, the film again is essentially formed at $t=8$ but the interface past $r=1.5$ shows much less deformation. As a consequence of their analysis, Maru et al. (1971) predict that film drainage will never be observed for $C a=C g=1$, while our results give strong indications to the contrary depending upon the viscosity ratio, $\lambda$.

The results of the constant-force cases described above demonstrate how the qualitative nature of the interface deformation changes relative to the constant velocity case. In both of the constant-force cases examined so far for $C g>\frac{2}{9}$, the dramatic decrease in sphere velocity (and thus of $\mathrm{Ca}$ and $\mathrm{Cg}$ ) results in the absence of long slender tails behind the sphere as were found in the case of constant velocity. To determine whether such a configuration is possible or if the deceleration of the sphere will always prohibit the formation of a long tail in creeping flow, a set of calculations was run duplicating an experiment of Maru et al. which produced a long tail behind the sphere, albeit at finite Reynolds number. Using a $3 \mathrm{~mm}$, glass sphere falling through cyclohexanol with a $62.5 \%$ glycerine-water solution as the fluid 1 


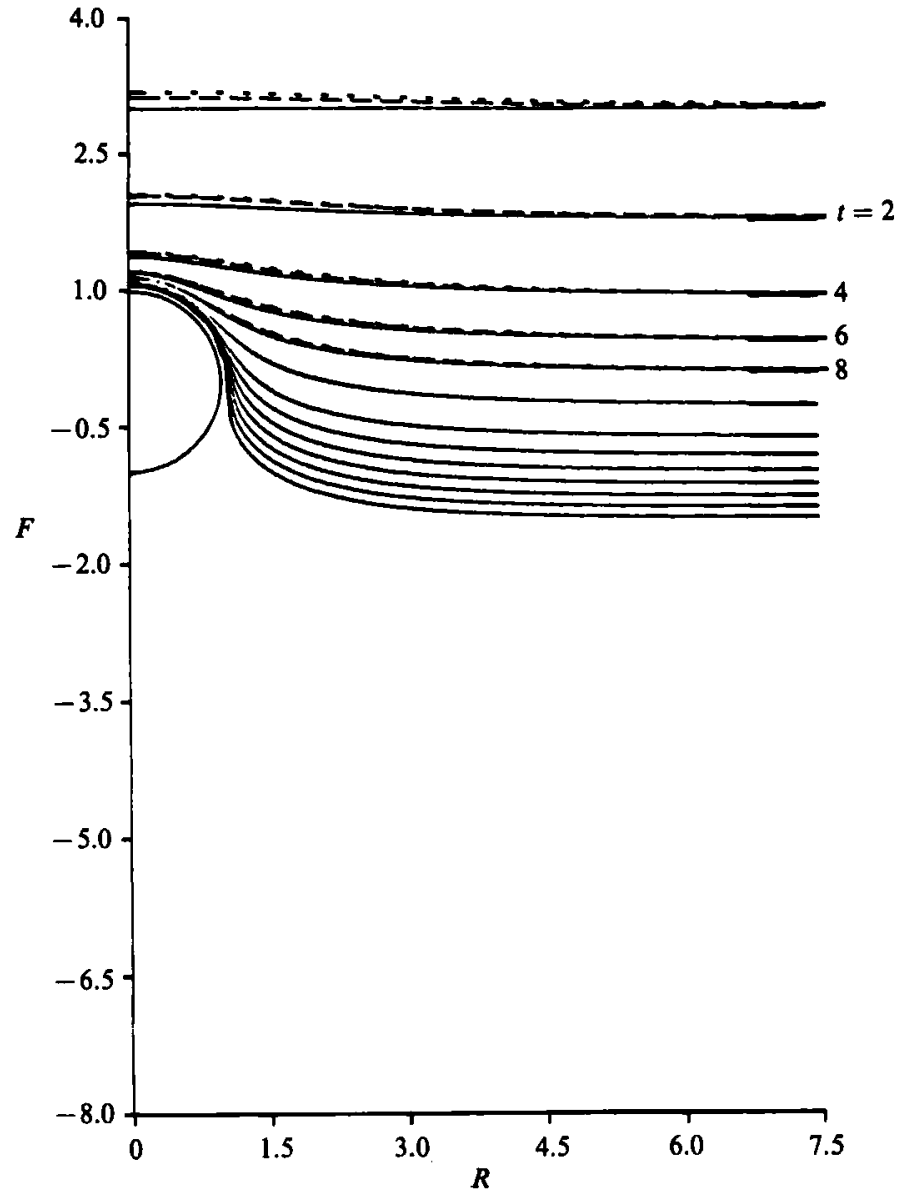

FIGURE 20. Interface shapes as a function of sphere position for $\lambda=10, C a=1, C g=1:-, l_{0}=3$; ,$-- l_{0}=5 ; \cdots, l_{0}=10$. Constant-force problem. Interface shown for $t=0,2,4,6,8,11,13,15$, $21,24,27,30,33$.

phase, Maru et al. observed a tail which reached a length of 120 sphere radii before exhibiting significant disturbances on its surface. The parameters of this system are $\lambda=0.434, C a=3.83, C g=1.97$; the Reynolds number is 15.19. With such a large Reynolds number, inertial effects are certainly not negligible, and it is by no means obvious that a long slender tail should be expected in the creeping-flow regime. However, figure 22 shows the calculated interface shapes versus position for this case and a long thin tail has very definitely developed. Figure 23 shows how the sphere velocity changes with position. Note that a minimum is observed at $l=-2.0$ (i.e. at $t=7.5$ ), which is related to the maximum found in the drag coefficient for motion for constant velocity. As the sphere becomes more encapsulated under the effect of surface tension, the velocity increases, eventually going above 1.00 , the value in an unbounded region of fluid 2 . The sphere would have a velocity of 2.304 in an unbounded domain of fluid 1 , but this velocity will never be achieved until breakthrough as the surrounding fluid 2 increases the density of the composite body above that of the sphere alone. The results of this calculation confirm the existence of a long slender tail for the case of a sphere moving under a constant buoyancy force. The comparison with published results can be of only a qualitative nature as no 


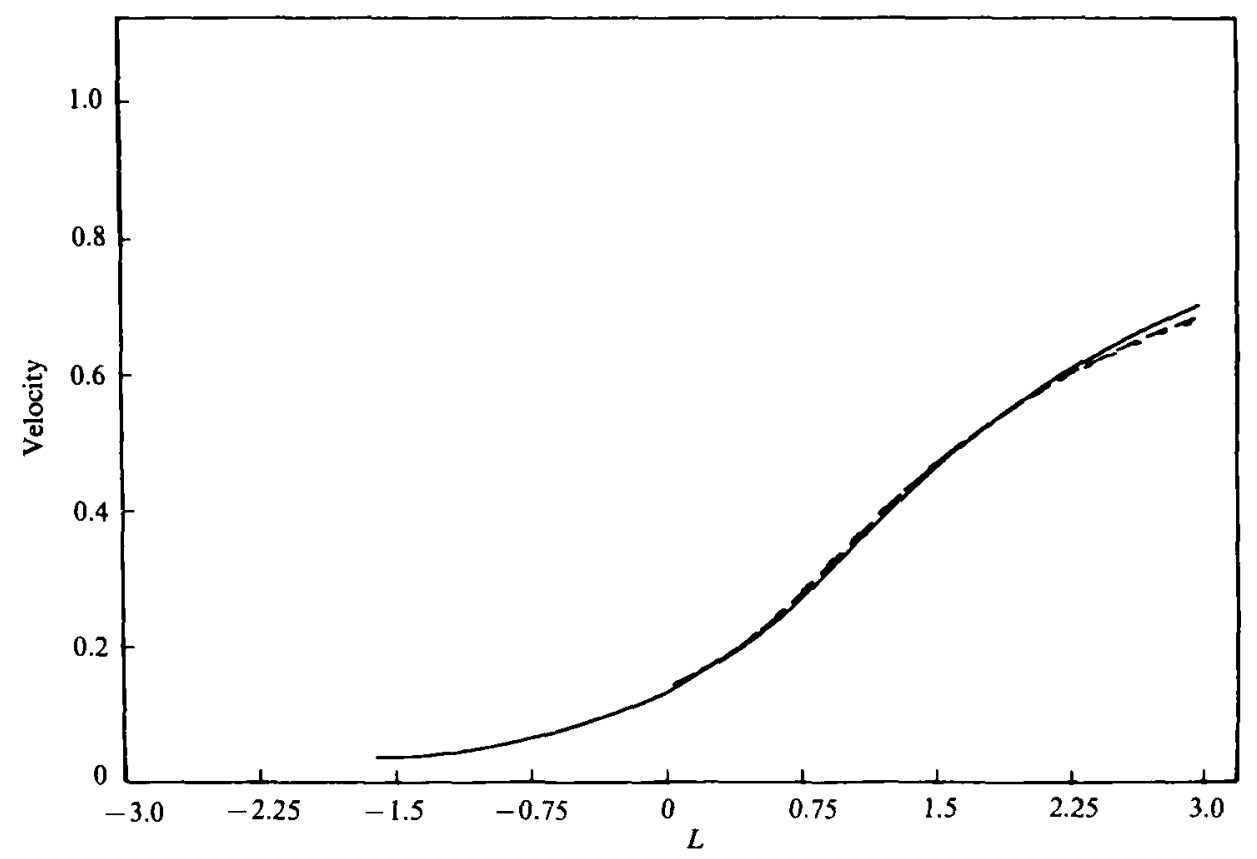

Fiqure 21. Velocity as a function of sphere position for $\lambda=10, C a=1, C g=1:-, l_{0}=3$; ,$-- l_{0}=5 ; \cdots,-\cdots, l_{0}=10$.

quantitative data on the interface shapes or sphere velocity were given by Maru et al. (1971).

As a further demonstration of the existence of tails in the constant-force problem, results for the case $\lambda=1, C a=1, C g=10$ are presented in figure 24 . This case again shows the definitive formation of a relatively long, slender tail. The velocity as a function of position is included in figure 23. At the minimum point, the velocity is approximately 0.4 times the Stokes velocity. Thus, $C a_{\text {eff }}$ and $C g_{\text {eff }}$ span the ranges $0.2-1$ and 2-10, respectively. The nearest constant-velocity would thus appear to be $\lambda=1, C a=C g=1$ and $\lambda=1, C a=10^{-1}, C g=1$. Indeed, the interface shows many characteristics of the constant-velocity results for the latter case. The broad deformation and surface-tension-driven constriction are both features which reappear in the constant-force result. The rapid constriction of the tail which occurred in the constant-velocity problem, however, is not present here. As the sphere continues past $l=-3$ and begins to accelerate, the interface behaves more like the $\lambda=1, C a=1$, $C g=1$ constant-velocity case. For this set of parameters, the surface-tension-driven constriction is decreased and this is reflected by the deceleration of pinching in the tail after $t=13$. Also, the increased viscous force is able to support the fluid in the tail.

The most interesting comparison, however, is with the results shown earlier for motion with a constant force at $\lambda=1, C a=C g=1$. The increase in $C g$ from 1 to 10 means that the effect of body forces on interface deformation should be relatively unimportant compared to capillary forces in the case $\lambda=1, C a=1, C g=10$, and the comparison with $\lambda=1, C a=C g=1$ further allows us to observe the way in which interfacial tension and density differences influence deformation. Comparing the results of figures 18 and 24 , the chief difference at early times when the sphere velocity is not too strongly influenced by the interface (so that the $C a_{\text {eff }}$ values are similar) 


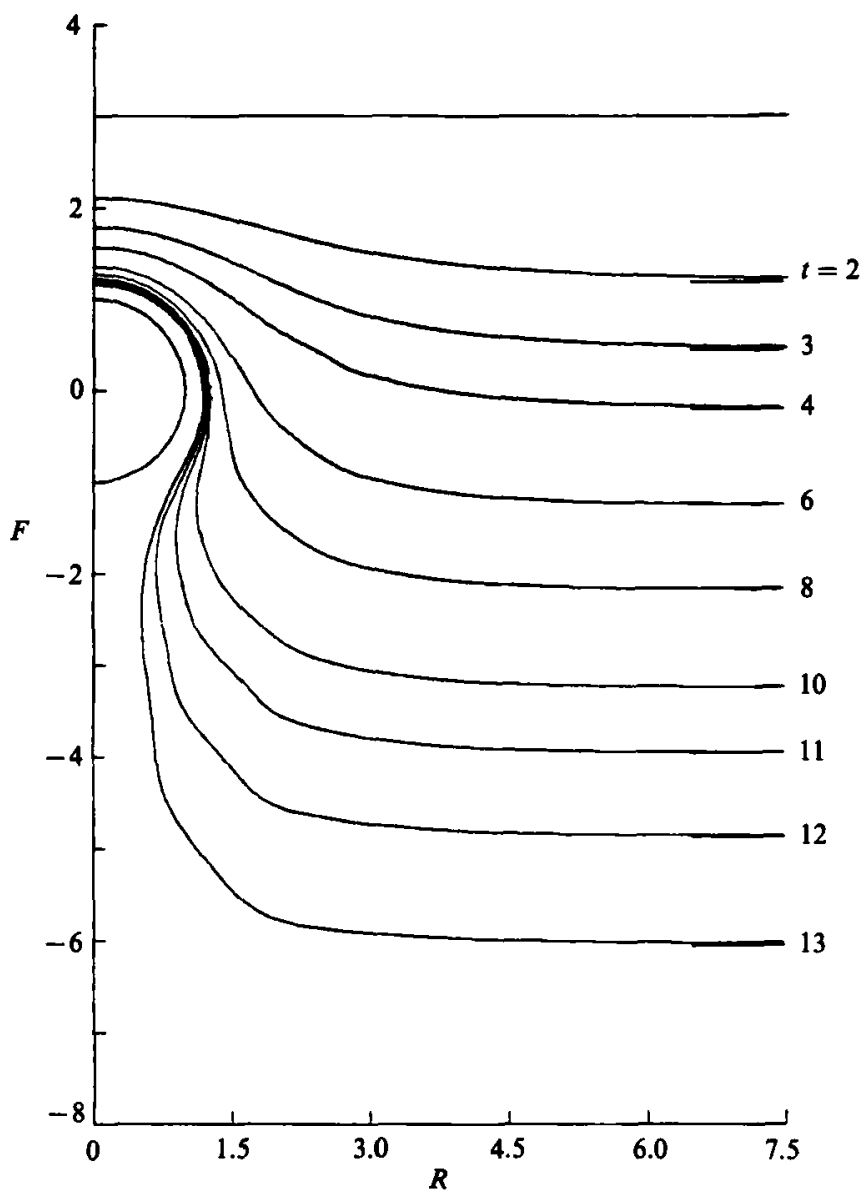

FiaUre 22. Interface shape as a function of sphere position for $\lambda=0.434, C a=3.831$, $C g=1.972, l_{0}=3$. Constant-force case.

is that the deformation produced is broader in the case $C g=10$. This is a consequence of the fact that the smaller density difference in this case yields less resistance to the tendency of interfacial tension to produce a broad, flat deformation, i.e. the system, all else being equal can support a larger volume of fluid 2 across the $(z=0)$-plane when $C g$ is increased.

As the sphere continues to move, an increasing volume of the heavy fluid is carried across the $(z=0)$-plane until finally, the force applied from the sphere can no longer support such a large volume of fluid, drainage accelerates and a tail configuration results. Obviously, for larger $C g$ values, a larger volume of fluid can be supported and this is clearly reflected when comparing results from figures 18 and 24, though comparison is difficult owing, in part, to the fact that the changes in sphere velocity are different in the two cases. As was true for the constant-velocity systems, once the tail configuration appears, the role of interfacial tension changes from one of tending to preserve the flat interface to one of minimizing the surface area in the tail region by producing a spherical shell around the sphere with the tail pinched off. This is demonstrated dramatically in the $\lambda=1, C a=1, C g=1$ results where the significantly lower value of $C a_{\text {eff }}$ from the case of figure 24 (0.1 vs. 0.4) causes a much more rapid pinching of the fluid in the tail region. 


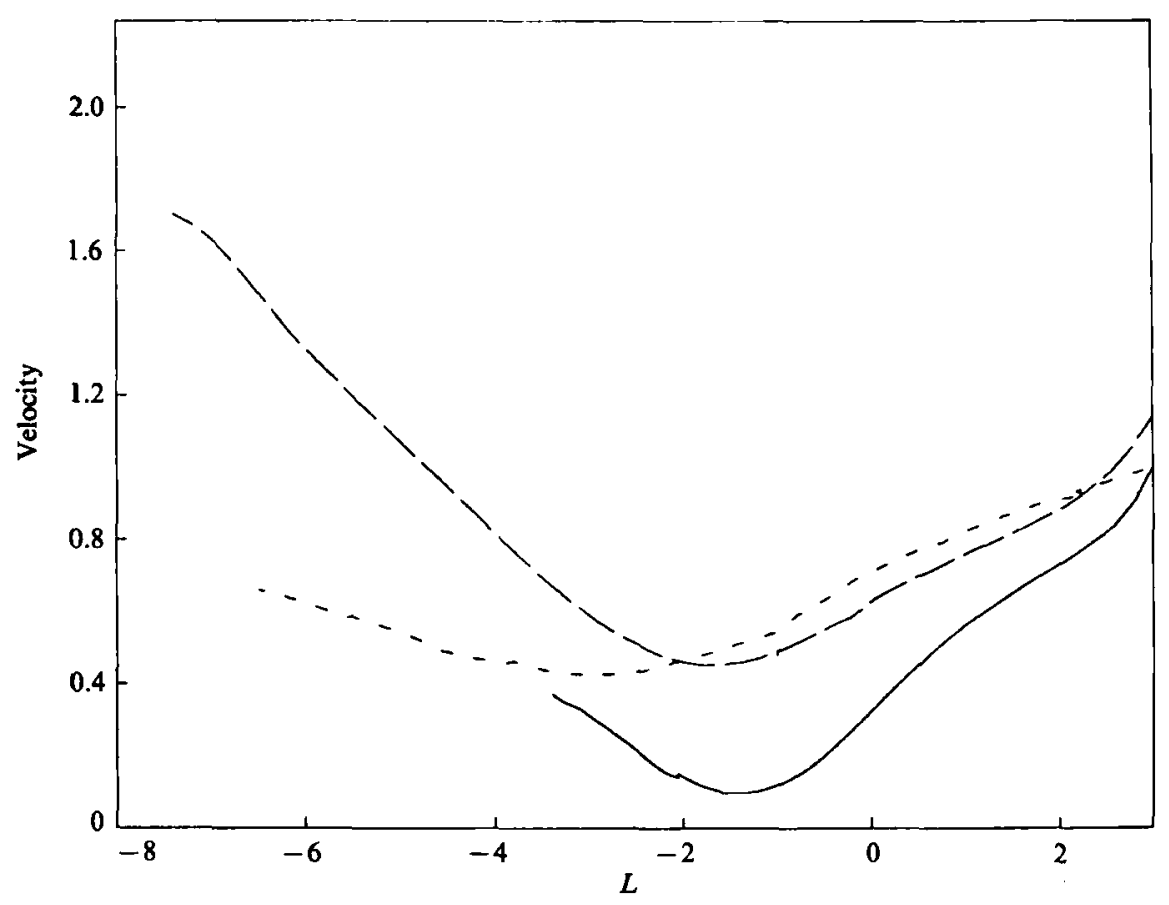

Fiadre 23. Velocity as a function of sphere position for constant-force cases.

\section{Effect of sphere starting position}

Previously, we have discussed the effect of starting positions greater than or equal to three sphere radii from the undeformed interface. These results were presented to show that if one is interested in the behaviour of the sphere-interface system some time after the sphere begins to approach the interface, results for all calculations begun at a distance greater than $l_{0}=3$ will appear identical. Here, we discuss another aspect of the initial-starting-point question : the effect of starting the sphere very close to the flat interface. Two groups of calculations have been carried out. In the first, a series of cases were run in which the sphere centre was initially 1.2 radii from a flat interface. In the second, initial starting positions were chosen so that the minimum initial gap between the sphere and a flat interface was comparable to the film thickness at large times when the initial position was $l_{0} \geqslant 3$. These second calculations were done only for sets of parameters which displayed tailing with a fairly stable film thickness in front of the sphere when started with $l_{0}=3.0$. This type of calculation more closely duplicates the experiments described in $\$ 1$ where a drop was brought close to an interface and then released, and may reveal cases where the starting position causes a change from the tailing configuration to one of film drainage (as was observed in all of the experiments other than Maru et al. 1971).

\subsection{Initial starting position, $l_{0}=1.2$}

Calculations with starting positions $l_{0}=1.2$ were performed for several cases. These included for constant velocity $\lambda=1, C a=C g=1.10$, a tailing case; and the constant force cases $\lambda=1, C a=C g=0.10$, and $\lambda=0.022, C a=0.465, C g=0.089$ which represent a tailing and a film-drainage configuration, respectively. In all of these 


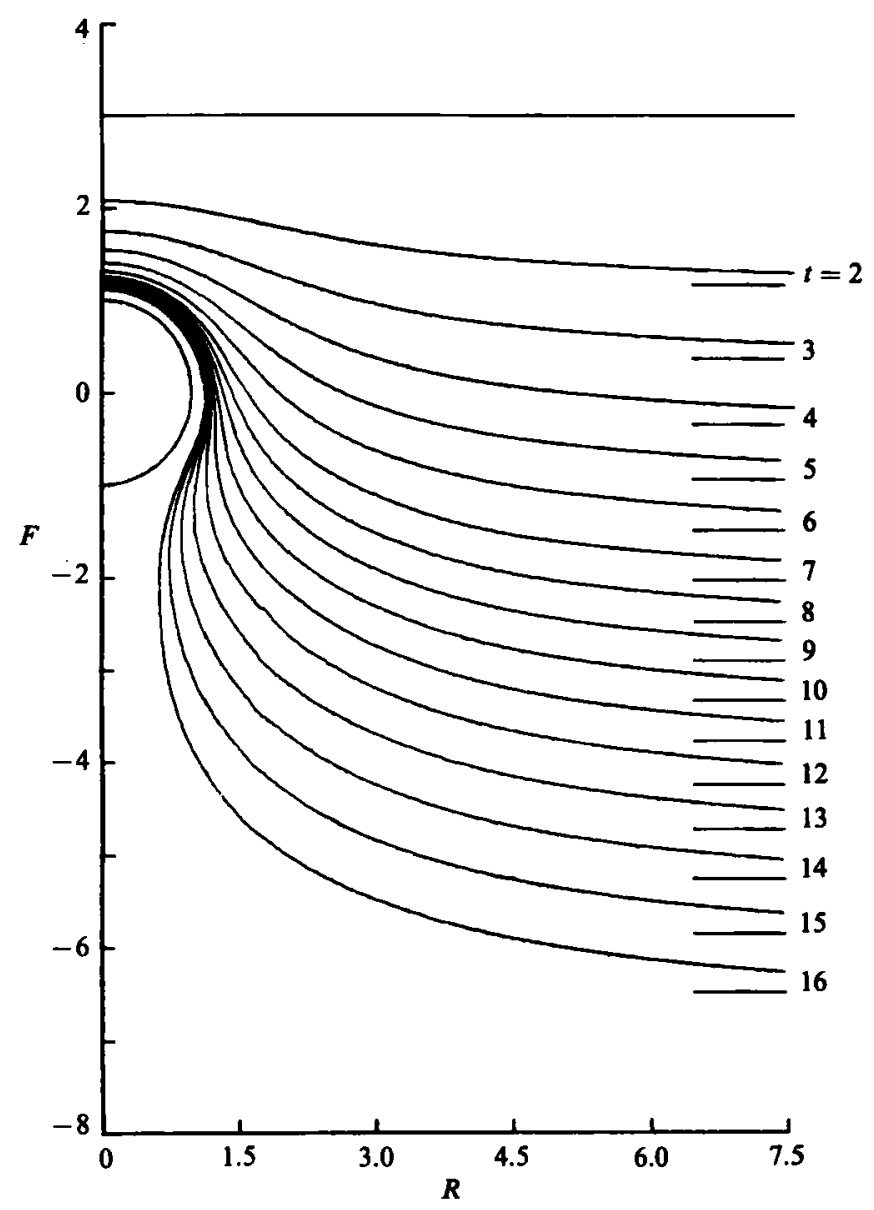

FIGURE 24. Interface shape as a function of sphere position for $\lambda=1, C a=1, C g=10, l_{0}=3$. Constant-force case.

cases, the calculated values for the velocities, stress and position of the interface at some value of $l$ came to within $1 \%$ of the values calculated when the initial position was 3.0. When this occurred, the calculations were stopped. The quasi-steady assumption made in deriving (13)-(16) ensures that for a given set of parameters, only the current position of the sphere and interface influence the future behaviour of the system; the prior history of the calculation will thus influence future results only through its effect on the current interface shape and sphere position. Therefore, when the positions of the sphere and interface in the $l_{0}=1.2$ calculation are the same as in the $l_{0}=3.0$ case, all future results will be identical as well. Figure 25 shows the $l_{0}=1.2$ results for $\lambda=1, C a=C g=0.10$ with a constant sphere velocity, and this may be compared to figure 9 which gives results for the same $\lambda, C a$ and $C g$ values, but $l_{0}=3.0$. The specific time it takes for the $l_{0}=1.2$ and $l_{0}=3.0$ interface shapes to become equal depends on the dimensionless parameters of the system; however, these results demonstrate that no change in the mechanism of breakthrough occurs when an initial gap thickness of 0.2 is used rather than 2.0 corresponding to the $l_{0}=3.0$ calculations. 


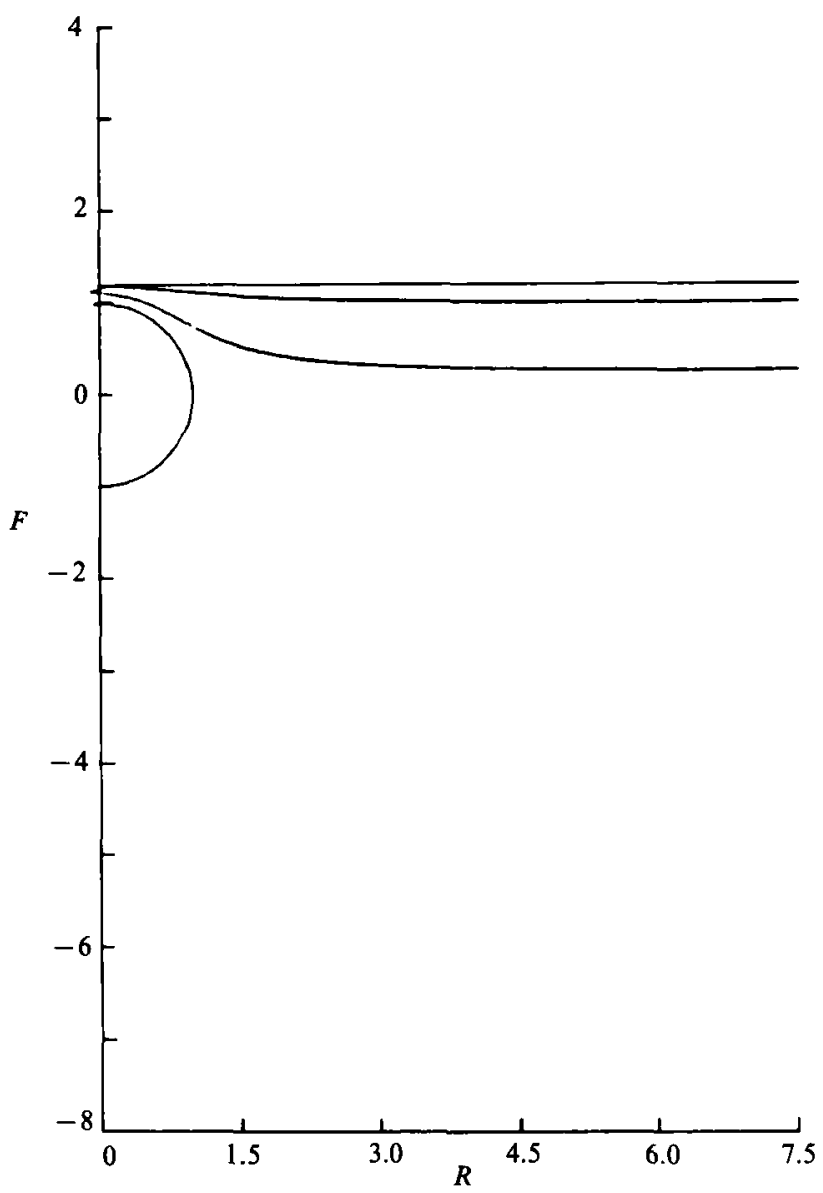

Figure 25. Interface shape as a function of sphere position for $\lambda=1, C a=C g=0.10, l_{0}=1.2$. Constant-velocity case.

6.2. Initial position, $l_{0}$, corresponding to the thickness of the film at large deformation This calculation differs significantly from all those discussed previously. In all the cases presented up to this point, film and tail formation first occurred simultaneously, then film drainage took place as the tail lengthened and narrowed. The present situation is one in which a thin film is present from the onset of the calculation, whereas the tail must still be formed from the flat interface. The implication of this in the constant-velocity problem is that the sphere may move across the interface before a tail has time to form. In the case of constant applied force, the probability of this happening is enhanced by the fact that the small gap between the sphere and interface will produce high velocity gradients right from the beginning of the calculation and so make a large contribution to the drag on the sphere. This results in a lower initial sphere velocity and a corresponding decrease in the effective values of $C a$ and $C g$. Small values for $C a_{\text {epf }}$ and $C g_{\text {eff }}$ tend to inhibit deformation of the flat interface and result in film drainage for breakthrough.

The constant-velocity case chosen was $\lambda=1.0, C a=C g=0.10$. For $l_{0}=3.0$, this case displays a short, pinched tail and narrow film surrounding the sphere. The fact that the tail is short suggests that tail formation is likely to be suppressed in going to the close starting position. The thickness of the film after formation of the tail 


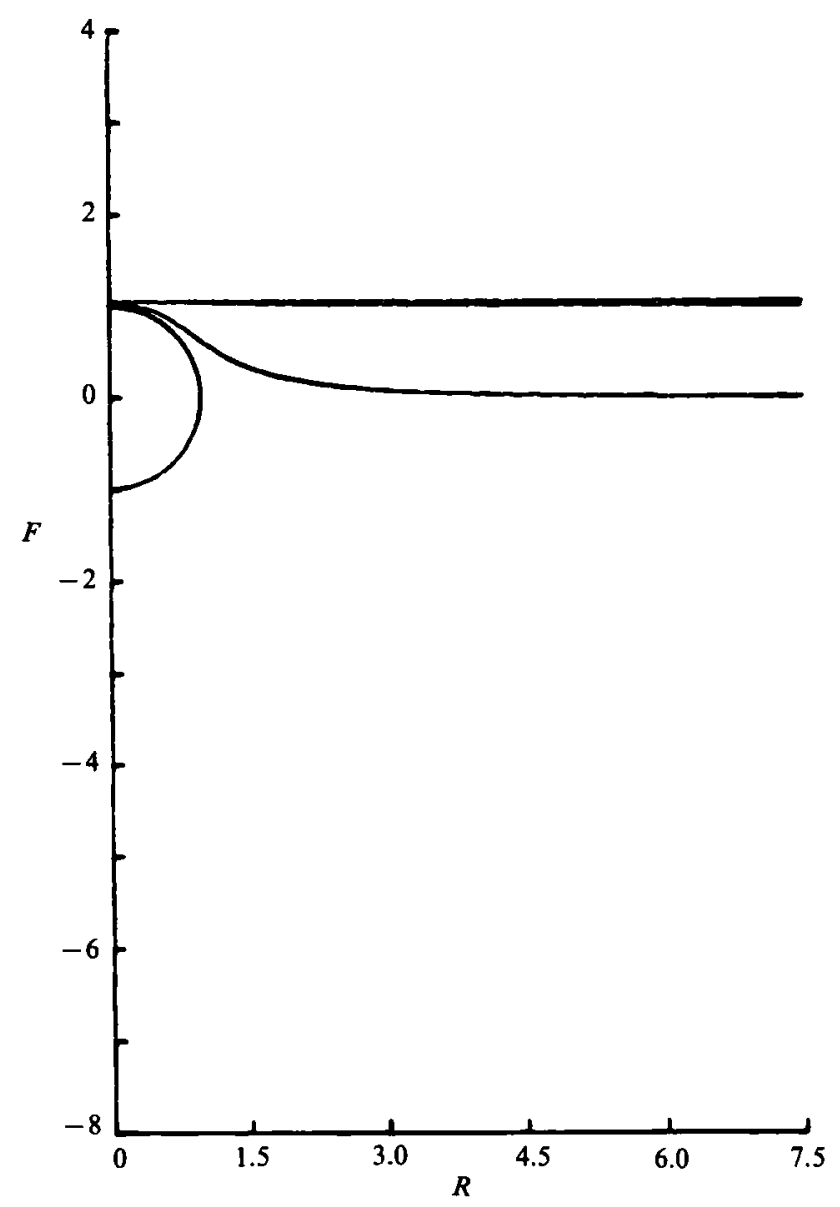

Figure 26. Interface shape as a function of sphere position for $\lambda=1, C a=C g=0.10$, $l_{0}=1.05$. Constant-velocity case.

remains relatively constant at 0.05 and this was the separation between the sphere and interface at the start of the calculation, i.e. $l_{0}=1.05$. Comparing figures 9,25 and 26 for $l_{0}=3,1.2$ and 1.05, respectively, it can be seen that the interface shapes are fairly similar; however, in the $l_{0}=1.05$ case, calculations would simply not converge much past $l=0$, i.e. the sphere straddling the interface, in spite of the fact that the convergence had been achieved in other cases with even thinner films between the sphere and interface. It is possible that no solution exists beyond the point of convergence for this case with $l_{0}=1.05$. In this case, the change in starting position of the sphere would have caused a change in the mode of breakthrough for $\lambda=1, C a=C g=0.1$. However, the solution technique utilized here does not allow a definitive case that the lack of convergence is not simply a failure of the numerics.

For a constant-force problem, the case of $\lambda=1, C a=1, C g=10$ was run with $l_{0}=1.10$ corresponding to the film thickness seen in the $l_{0}=3.0$ result. The results of this calculation are shown in figure 27 . One sees that the two cases agree in the film region but the results for $l_{0}=1.1$ show a narrower tail than for the $l_{0}=3.0$ result. Also, although a tail is formed, the total deformation of the interface in the $l_{0}=1.10$ case is much less than for the large starting distance. That is, the interface for $l_{0}=1.10$ lies below that for $l_{0}=3.0$ at equal sphere positions. The result of starting from 


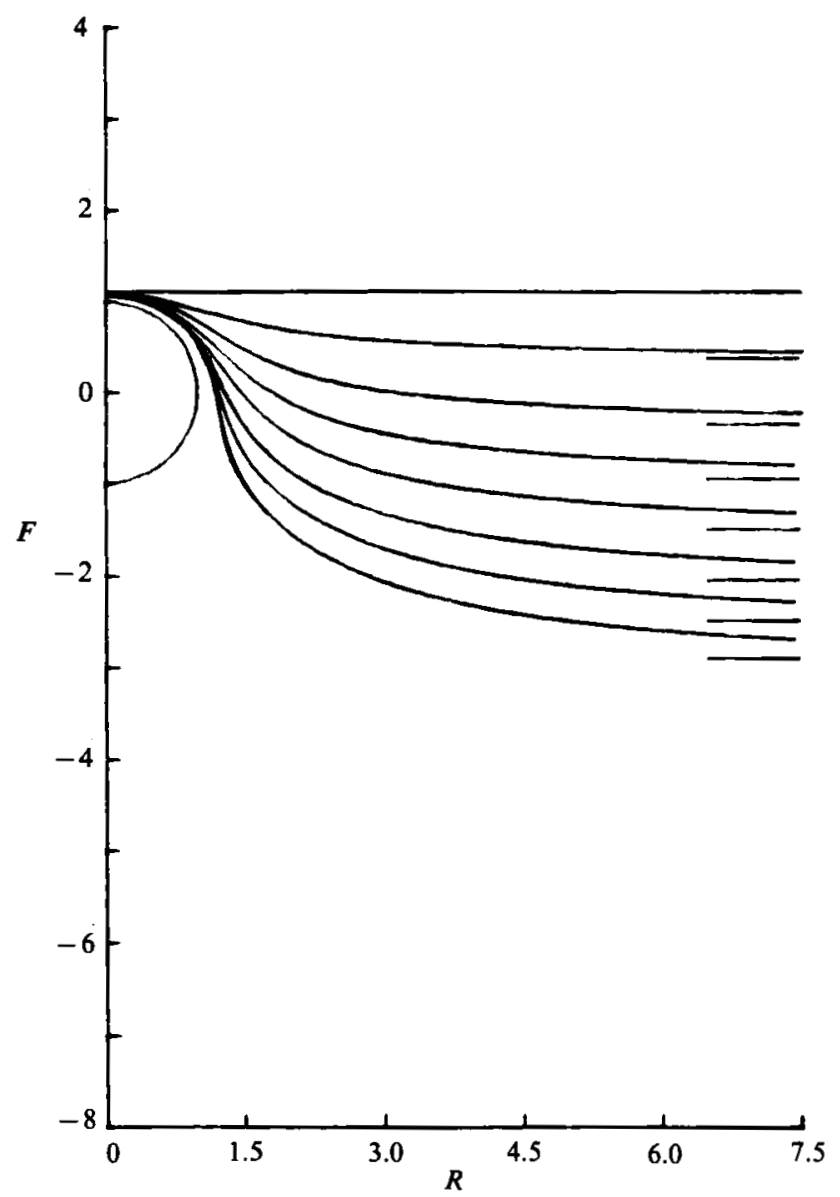

Fiaure 27. Interface shape as a function of sphere position for $\lambda=1, C a=1, C g=10$, $l_{0}=1.10$. Constant-velocity case.

$l_{0}=1.10$ is that tailing is enhanced in the constant-force case and so rather than changing the mode of breakthrough, the close starting positions hasten breakthrough in the existing mode.

The effect of starting the sphere close to the interface may be summarized by stating that the initial conditions influence the deformation for some time after the calculation is begun. For the case when $l_{0}=1.2$, the departure of the calculation from the $l_{0}=3.0$ result diminishes with time until the results are identical. For a starting position corresponding to the film thickness after a tail has been established, the departure from the $l_{0}=3.0$ result may be significant enough to cause a change in the mode of breakthrough for the constant-velocity case $\lambda=1, C a=C g=0.10$, and does cause a departure in the interface shape for the constant-force case $\lambda=1, C a=1$, $C a=10$ for a long time after the calculation is started.

\section{Conclusions}

We have used the boundary-integral to study numerically the creeping axisymmetric motion of a sphere normal to an initially flat fluid-fluid interface under the constraint that the sphere moves either with a constant velocity or under the action of a constant-buoyancy force. 
Our calculations show the existence of two distinct modes of interface deformation for the constant-force problem which we have designated as film draining and tailing. The parameters controlling which mode will occur are $C a$ and $C g$, reflecting the relative magnitudes of the interfacial tension and the density difference between the two fluid phases; the forces associated with these two properties tend to maintain the interface in its initially flat configuration. Thus, when these restoring forces are large relative to the viscous forces generated by the motion of the sphere, i.e. $C a, C g \ll 1$, film-drainage behaviour occurs. When the inverse is true, i.e. $C a, C g$ approximately order 0.1 or greater, a tailing configuration appears. A further statement about the possibility of tailing for the constant-buoyancy-force case can be made : no tails can occur when the density of the sphere is intermediate to those of the two fluids.

A significant difference in the behaviour of the constant-force and constant-velocity problems is the shortening of the tails in the former case for moderate $C a$ and $C g$. This follows from the decrease of the instantaneous effective deforming forces as the sphere approaches the interface. As the sphere comes closer to the interface, its velocity decreases, thereby increasing the strength of interfacial tension and density body forces due to the density difference relative to viscous forces. The increased relative strength of the restoring forces makes further deformation more difficult, with the final outcome being the suppressed extension of the tail and more film drainage occurring between the sphere and interface. The viscosity ratio acts not to restore the interface to its initial flat shape, but to control the rate at which fluid drains from the space between the sphere and interface.

Comparison of our results with published experimental data showed fair agreement. The differences which do exist are believed to be a consequence of the differences in the initial conditions used, but as the experimental descriptions did not state these initial conditions, this area cannot be explored at present. Calculations using small interface-sphere separations showed that changes in initial conditions could significantly influence results, especially at early times.

\section{Appendix : Macroscopic force balance for a sphere several radii beyond an initially undeformed interface}

To prove that a sphere moving under a constant body force cannot cross the plane of the initially undeformed interface when the body force is gravity alone, a macroscopic force balance is carried out for a sphere which is assumed to have crossed the plane $z=0$, carrying a body of fluid 2 with it in the form of a film plus tail. We shall see that a contradiction is reached in this balance if the density of the sphere is between that of the two fluids, thus showing that a configuration of the tailing type is impossible for this case.

We begin by stating the force balance for the sphere alone

$$
F_{\mathrm{ext}}-\rho_{\mathrm{s}} g V_{\mathrm{s}}-\int_{S_{\mathrm{P}}}\left(\boldsymbol{T}_{2} \cdot \boldsymbol{n}\right) \cdot \boldsymbol{k} \partial S_{\mathrm{P}}+\rho_{2} g V_{\mathrm{s}}=0,
$$

where $\rho_{2}$ is the density of the fluid in which the sphere is immersed.

$\boldsymbol{T}_{2}$ is the dynamic stress tensor in fluid 2 (i.e. the total stress minus hydrostatic pressure contributions);

$n \quad$ is the outer unit normal to the sphere;

$\boldsymbol{k}$ is a unit normal in the vertical (z) direction;

$S_{\mathbf{P}} \quad$ is the volume of the sphere, ${ }_{3}^{4} \pi a^{3}$;

$F_{\text {ext }} \quad$ is any external force on the sphere in addition to gravity. 


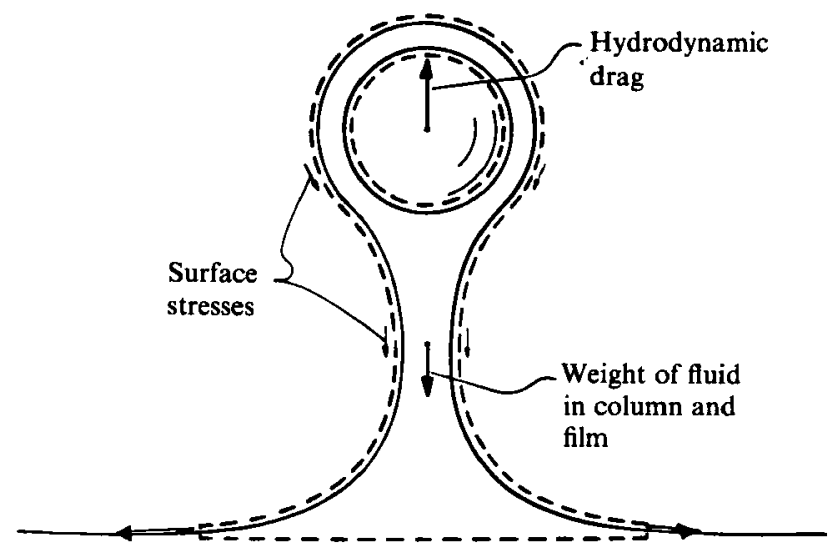

Figure 28. Schematic sketch of control volume for an overall force balance on the fluid in the tail plus film.

A similar balance on the fluid region (control volume) marked inside the dashed lines of figure 28 gives

$$
\rho_{1} g V_{\mathrm{t}}-\rho_{2} g V_{\mathrm{t}}+\int_{S_{\mathrm{P}}}\left(\boldsymbol{T}_{2} \cdot \boldsymbol{n}\right) \cdot \boldsymbol{k} \partial S_{\mathrm{P}}-\rho_{2} g V_{\mathrm{s}}+\int_{S_{\mathrm{I}}}\left(\boldsymbol{T}_{1} \cdot \boldsymbol{n}\right) \cdot \boldsymbol{k} \partial S_{\mathrm{I}}=0,
$$

where

$V_{\mathrm{f}}$ is the volume of fluid 2 in the tail plus film region inside the dashed lines of figure 4;

$V_{\mathrm{t}}$ is the sum of $V_{\mathrm{f}}$ and $V_{\mathrm{s}}$

$\boldsymbol{T}_{1}$ is the dynamic stress tensor in fluid 1 ;

$S_{I}$ is the portion of the surface of the control volume defined by the interface between fluids 1 and 2 ;

$S^{*}$ is the portion of the surface of the control volume which is coincident with the initial plane of the interface.

If we neglect the contribution due to $T_{2}$ at $S^{*}$, and assume $\lambda=0$ so that $\dagger$

$$
\int_{S_{\mathrm{I}}}\left(\boldsymbol{T}_{1} \cdot \boldsymbol{n}\right) \cdot \boldsymbol{k} \partial S_{\mathrm{I}}=0
$$

the balance (1) and (2) can be combined to yield

$$
\frac{V_{\mathrm{t}}}{V_{\mathrm{s}}}=\frac{\rho_{2}-\rho_{\mathrm{s}}}{\rho_{2}-\rho_{1}}+\frac{F_{\text {ext }}}{g\left(\rho_{2}-\rho_{1}\right) V_{\mathrm{s}}} .
$$

We consider the case $F_{\text {ext }}=0$ in which the only 'external' force on the sphere is due to buoyancy. Now, it is obvious that $V_{\mathrm{t}} / V_{\mathrm{s}}>1$. On the other hand $\left(\rho_{2}-\rho_{\mathrm{s}}\right) /\left(\rho_{2}-\rho_{1}\right)<1$ unless $\rho_{\mathrm{s}}<\rho_{1}$. Hence, we conclude that the configuration represented by figure 28 is impossible unless $\rho_{\mathrm{s}}<\rho_{1}$. No such inconsistency arises if we apply the same force balance concepts to a film-drainage configuration with $\rho_{1}<\rho_{2}$. The presence of viscosity in fluid $1, \lambda \neq 0$, would act to slow the drainage of the fluid in the tail, but could not provide a force to actually overcome gravity. Therefore, the conclusions from (A 3) would hold even for a viscous fluid 1.

$\dagger$ The mass flux at $S^{*}$ is seen to be small after calculating the change in tail volume with time. Furthermore, the velocity and velocity gradients in this region are also small. 


\section{REFERENCES}

Berdan, C. 1982 Ph.D. dissertation. California Institute of Technology.

Berdan, C. \& Leal, L. G. 1982 Motion of a sphere in the presence of a deformable interface. Part 3: Numerical study of the translation of a sphere parallel to an interface. $J$. Colloid Interface Sci. 87, 62.

Chin, H. B. \& HAN, C. D. 1979 Studies on droplet deformation and breakup. I. Droplet deformation in extensional flow. J. Rheol. 23, 557.

GELLER, A.S. 1986 A study of the creeping motion of a sphere normal to a deformable fluid interface: deformation and breakthrough. Ph.D. dissertation. California Institute of Technology.

Grace, H. P. 1971 Eng. Foundation 3rd Res. Conf. on Mixing. Andover, NH.

Hartland, S. 1968 The approach of a rigid sphere to a deformable liquid/liquid interface. J. Colloid Interface Sci. 26, 383.

HaRTLAND, S. 1969 The profile of a draining film between a rigid sphere and a deformable fluid-liquid interface. Chem. Engng Sci. 24, 987.

Jeffreys, G. V. \& Davies, G. A. 1971 In Recent Advances in Liquid/Liquid Extraction (ed. C. Hanson), p. 495, Pergamon Press.

Johnson, R. E. \& SADHaL, S.S. 1985 Fluid mechanics of compound multiphase drops and bubbles. Ann. Rev. Fluids 17, 289.

Jones, A. F. \& WILson, S. D. R. 1978 The film drainage problem in droplet coalescence. J. Fluid Mech. 87, 263.

KirkPatric, R. D. \& LocketT, M. J. 1974 The influence of approach velocity on bubble coalescence. Chem. Engng Sci. 29, 2363.

LANG, S. B. \& WILKE, C. R. 1971 A hydrodynamic mechanism for the coalescence of liquid drops. I. Theory of coalescence at a planar interface. I. and E. C. Fundamentals 10, 329.

Ladyzhenskaya, O. A. 1963 The Mathematical Theory of Viscous Incompressible Flow. Gordon and Breach.

Leal L. G. \& LEe S. H. 1981 Proceedings of IUTAM-IUTPAC Symposium on Interactions of Particles in Colloid Dispersions, March 16-21. Canberra.

Lee, S. H., Chadwick, R. S. \& Leal, L. G. 1979 Motion of a sphere in the presence of a plane interface. Part 1 : An approximate solution by generalization of the method of Lorentz. J. Fluid Mech. 93, 705.

LeE, S. H. \& Leal, L. G. 1980 Motion of a sphere in the presence of a plane interface. Part 2: An exact solution in bipolar coordinates. J. Fluid Mech. 98, 193.

LEE, S. H. \& LEAL, L. G. 1982 Motion of a sphere in the presence of a deformable interface. Part 2: Numerical study of the translation of a sphere normal to an interface. J. Colloid and Interface Sci. 87, 81.

MARU, H. C., WASAN, D. T. \& KintneR, R. C. 1971 Behavior of a rigid sphere at a liquid-liquid interface. Chem. Engng Sci. 26, 1615.

Mikami, T., Cox, R. G. \& Mason, S. G. 1975 Breakup of extending liquid threads. Intl J. Multiphase Flow 2, 113.

Narayaran, S., Gossens, L. H. J. \& Kossen, N. W. F. 1974 Coalescence of two bubbles rising in line at low Reynolds number. Chem. Engng Sci. 29, 2071.

Olbricht, W. L. \& LeaL, L. G. 1983 The creeping motion of immiscible drops through a converging/diverging tube. J. Fluid Mech. 134, 329.

Princen, H. M. 1963 Shape of a fluid drop at a liquid-liquid interface. J. Colloid Sci. 18, 178.

RAYLEIGH, LORD 1892 On the instability of a cylinder of viscous liquid under capillary force. Phil. Mag. 34, 145.

Shah, S. T., Wasan, D. T. \& Kintner, R. C. 1972 Passage of a liquid drop through a liquid-liquid interface. Chem. Engng Sci. 27, 881.

SMIth, P. G. \& VAN DE VEN, T. G. M. 1984 The effect of gravity on the drainage of a thin liquid film between a solid sphere and a liquid/fluid interface. J. Colloid Interface Sci. 100, 456.

ТомотікA, S. 1936 Breaking up of a drop of viscous liquid immersed in another viscous fluid which is extending at a uniform rate. Proc. $R$. Soc. Lond. A 153, 302. 